\title{
Secondary-electron-emission spectroscopy of tungsten: Angular dependence and phenomenology
}

\author{
Willis, Roy F.; Christensen, Niels Egede
}

Published in:

Physical Review B

Link to article, DOI:

10.1103/PhysRevB.18.5140

Publication date:

1978

Document Version

Publisher's PDF, also known as Version of record

Link back to DTU Orbit

Citation (APA):

Willis, R. F., \& Christensen, N. E. (1978). Secondary-electron-emission spectroscopy of tungsten: Angular dependence and phenomenology. Physical Review B, 18(10), 5140-5161.

https://doi.org/10.1103/PhysRevB.18.5140

\section{General rights}

Copyright and moral rights for the publications made accessible in the public portal are retained by the authors and/or other copyright owners and it is a condition of accessing publications that users recognise and abide by the legal requirements associated with these rights.

- Users may download and print one copy of any publication from the public portal for the purpose of private study or research.

- You may not further distribute the material or use it for any profit-making activity or commercial gain

- You may freely distribute the URL identifying the publication in the public portal

If you believe that this document breaches copyright please contact us providing details, and we will remove access to the work immediately and investigate your claim. 


\title{
Secondary-electron-emission spectroscopy of tungsten: Angular dependence and phenomenology
}

\author{
Roy F. Willis \\ Surface Physics Group, Astronomy Division, European Space Research and Technology Center, Noordwijk, Holland
}

Niels Egede Christensen

Physics Laboratory I, The Technical University of Denmark, 2800 Lyngby, Denmark

(Received 1 August 1977; revised manuscript received 23 June 1978)

\begin{abstract}
Angle-resolved energy-distribution measurements of secondary-electron emission (SEE) from metals reveal spectral fine structure that relates directly to the density distribution of the one-electron states throughout $E-\overrightarrow{\mathrm{K}}$ space located above the vacuum level $E_{v}$. The angular dependence of the SEE spectra from (100), (110), and (111) tungsten surfaces has been studied as a function of polar angle $0^{\circ} \leqslant \Theta \leqslant 70^{\circ}$ along azimuthal directions $\phi$ such that the energy- and angle-resolved SEE current $j_{\mathrm{SEE}}(E, \Omega)$ effectively scans states throughout the $1 / 48$ th irreducible body-centered-cubic zone. Calculations have been carried out in both "reduced" and "extended" $\vec{K}$ space in order to assess the relative contribution of elastic umklapp scattering to the density distribution of contributing states profiles. The results indicate that the overall secondary-electron yield may be represented as the sum of basically two contributions $J_{\mathrm{SEE}}^{\text {total }}=$ $\int_{0}^{\pi} d \Omega \int_{0}^{E_{\max }} j_{\mathrm{SEE}}(E, \Omega) d E=J_{\mathrm{SEE}}^{\text {bulk }}+J_{\mathrm{SEE}}$ surface. The bulk contribution represents emission due to Bloch waves propagating out of states in the semi-infinite crystal; the surface contribution represents that part of the current due to evanescent waves at the metal-vacuum interface. In addition, transmission-induced spectral features are observed (transmission resonances), which are not related to the density-of-states fine structure, but are due to a quantum-mechanical enhancement in the escape probability arising from wave-function matching at the emitter-vacuum interface. Bulk and surface band-structure effects are concurrently manifest in the SEE spectra via the wave-matching conditions imposed at the solid-vacuum interface. The results are discussed within the general conceptual framework provided by "the (time-reversed) incoming final-state wavefunction" approach to electron emission phenomenology of metal surfaces, thereby establishing a relationship with recently developed low-energy electron diffraction, photoemission, and field-emission formalism.
\end{abstract}

\section{INTRODUCTION}

The phenomenon of secondary-electron emission (SEE) from metals has been studied extensively for many years. ${ }^{1}$ However, relatively few measurements of the angular variation of the energy distribution of secondary electrons from singlecrystal surfaces have been reported in the voluminous literature on this subject. This may be ascribed in part in the past to the experimental difficulties inherent in such measurements, but mainly due to the apparent absence of any particularly interesting or noteworthy spectral features worthy of more detailed study.

For example, one of the earliest studies by Jonker ${ }^{2}$ who recorded the angle-resolved energy distribution of the secondary current $j_{\mathrm{SEE}}(E, \Omega)$ for a series of emergent angles $\Omega$ ( $\Omega$ being defined in terms of the usual azimuthal angle $\phi$ and polar angle $\theta$ ), showed that secondary-electron emission from nickel exhibited a featureless cosine angular dependence. Later studies, however, notably by Burns $^{3}$ and Appelt ${ }^{4}$ [on $\mathrm{Ni}(100)$ and $\mathrm{Cu}(100)$ (Ref. 3), and $\mathrm{Cu}(110)$ (Ref. 4)], produced angular variations that differed significantly from cosine behavior. Attempts were made to interpret these results in terms of diffraction effects in directions defined by reciprocal-lattice $\vec{G}$ vectors supporting inter- band transitions. The energy resolution in these early experiments $(\Delta E \simeq 5$ to $10 \mathrm{eV}$ ) however, was insufficient to resolve any fine structure in the energy-distribution curves. The spectra showed a featureless continuum of secondaries that peaked strongly at low kinetic energies $\left(0 \lesssim E_{\mathrm{kin}} \lesssim 50 \mathrm{eV}\right)$. Quantum-mechanical calculations ${ }^{5-9}$ of the collision process between the incident electrons and those in the solid predicted the overall "featureless shape" of the secondary "cascade" energy-distribution curve in agreement with these early experimental spectra.

Subsequent measurements employing both hemispherical $^{10}$ and angle-selective ${ }^{11}$ analyzers with increased energy resolution $(\Delta E \lesssim 0.5 \mathrm{eV})$ succeeded in revealing weak spectral fine structure, which could not be attributed to Auger relaxation processes involving inner core levels. ${ }^{12}$ While it was recognized that such effects related to the bulk electronic band structure, it was not possible to establish their nature on the basis of these measurements and energy-band diagrams existing at that time.

An important source of secondary electrons arises from electron-hole pair interband transitions produced via the screened-Coulomb interaction between the incident (and subsequently excited electrons) and the valence electrons. ${ }^{6-8}$ 
We have demonstrated in a series of earlier papers for graphite crystals, ${ }^{13,14}$ that scattering out of unbound Bloch states located above the vacuum level $E_{v}$ gives rise to spectral-intensity variations which relate directly to the single-particle density of "unfilled" electronic states. These measurements were taken using a retarding-field hemispherical analyzer which recorded the energy distribution of the emitted current over a $180^{\circ}$ solid angle, $\int_{0}^{\pi} j_{\mathrm{SEE}}(E, \Omega) d \Omega$. Subsequent measurements using a small angle-selective $\left(0.1^{\circ}\right)$ analyzer ${ }^{15}$ revealed that spectra measured in directions normal to the low-index (100), (110), and (111) faces of tungsten single crystals show increased spectral definition of fine structure, which is closely related to the one-dimensional density-of-states function along the symmetry lines corresponding to each face, entirely analogous to similar directional photoemission studies conducted earlier..$^{16}$ The geometry (symmetry-oriented normal emission) is such that the component of the final-state wave vector parallel to the surface is negligibly small, $\overrightarrow{\mathrm{K}}_{\| 1} \rightarrow 0$, which assuming specular elastic (coherent) scattering boundary conditions, remains unchanged during transmission through the interface.

It is important to realize however that, due to refraction, the above SEE spectral fine structure ${ }^{15}$ only relates to the one-dimensional density of states, along a specific line in $\overrightarrow{\mathrm{K}}$ space, when the symmetry direction is normal to the emitting surface. ${ }^{17}$ Although the actual experiment might refer to a specific direction of the emitted current, $j_{\mathrm{SEE}}(E, \Omega)$, angle-resolved measurements in offsymmetry directions contain contributions to each element of spectral structure from quite general points (and often several bands) located throughout $\overrightarrow{\mathrm{K}}$ space, i.e., it is rarely the case that any single feature can be identified with any single contribution from any single band, particularly at the higher $\left(E_{K} \geqslant 10 \mathrm{eV}\right)$ energies. This implies that calculations relating to a detailed high-energy band structure must be carried out in extended $\overrightarrow{\mathrm{K}}$ space in order to avoid erroneous interpretation in terms of, for example, possible band dispersion effects associated with individual spectral peak shifts. From this point of view, angle-resolved SEE spectroscopic analysis is extremely pertinent to final-state effects in angle-resolved photoemission spectra..$^{18}$

In this paper, the experimental work described in Ref. 15 has been extended, and spectra for directions which are not normal to the three lowindex planes of tungsten are reported. The most commonly measured angular plot is of current versus polar angle $\theta, j_{\mathrm{SEE}}(E, \theta)$, for one value of azimuthal angle $\phi$. This is the approach we have adopted in this work. The angle-resolved measurements on the W (100), (110), and (111) faces effectively scan along azimuths $\phi$ which define states throughout the $\frac{1}{48}$ irreducible bcc zone. In order to examine whether the correspondence between the bulk energy bands and the measured SEE spectra, indicated in the earlier work, ${ }^{15}$ remains valid in general, it has been necessary to extend an existing band calculation ${ }^{19}$ to energies high above the Fermi level $E_{f}$, and from this derive theoretical distributions of contributing states. Extensive calculations,${ }^{20}$ which assume the component of momentum parallel to the surface to be conserved during emission, have been carried out in both the reduced- and extended-zone scheme. The object was to establish under what conditions umklapp elastic scattering from directions in $\overrightarrow{\mathrm{K}}$ space other than the direction of measurement projected into the first Brillouin zone contribute to $j_{\mathrm{SEE}}(E, \theta)$. Also, the calculations were able to identify the origins $(E, \vec{K})$ of the various contributions of the bulk bands to the SEE spectral structure. It follows from this comparison between the experimental spectra and the distribution of states predicted by the bulk energy-band calculations that any other effects, such as emission from surface states (resonances), etc., will show up as "anomalous features" in the measurements.

The tungsten energy-band structure above the vacuum level $E_{\text {vac }}$ is distinguished by a band gap located around $9 \mathrm{eV}$ above the Fermi level $E_{f}$ which extends (almost completely) throughout the Brillouin zone. This feature is clearly resolved as a characteristic intensity minimum in the SEE spectra from all three faces..$^{15}$ "Anomalous" spectral structure appears in this band-gap region (band-gap emission) identified with two sources: (a) a band of surface resonances intrinsic to the $\mathrm{W}(100)$ face with reduced surface wave vectors $k_{\|}$ extending over a large range of the associated surface Brillouin zone; and (b) "surface emission" due to the fact that the plane-wave vacuum states can always "tail" a short way into the solid (the wave-function amplitude decaying exponentially into the surface). A third source of anomalous structure, which is particularly pronounced in emission from $\mathrm{W}(111)$, but also occurs for $\mathrm{W}(110)$ and, in a specific instance for $W(100)$, is (c) that due to "transmission resonances" which relate to the dispersion behavior of the bulk energy bands and the wave-matching conditions imposed by the surface lattice. Enhanced emission occurs which produces spectral maxima not related to densitiesof-states fine structure.

Transmission-induced structure will clearly complicate any analysis of electron emission spectra based solely on density-of-states arguments. 
The quantum-mechanical formulation of the energy and $\overrightarrow{\mathrm{K}}$ dependence of the transmission probability function is by no means a trivial task. Recent developments, however, particularly in photoemission $^{21,22}$ and field-emission theory, ${ }^{23}$ have indicated that the problem effectively reduces to that of evaluating the transmission (scattering) matrix of an electron, with the same energy and wave vector in the surface, incident (in a time-reversed sense) on the metal surface from the vacuum side, i.e., determining the incident and scattered currents associated with a final-state wave function identical to that employed in low-energy electron-diffraction (LEED) theory. This time-reversed "incoming" final-state wave-function approach has shown quite clearly" that the conventional "threestep model"-excitation, transport to, and escape through the surface-used in almost all approaches to secondary-electron emission to date,$^{5-9}$ is strictly valid only under certain limiting conditions. It will be shown that the ramifications of this deeper conceptual understanding of electron emission from solids are directly applicable (and indeed necessary) for a full understanding of the angular-dependent SEE spectral intensities.

The aim of the present paper has been to relate the observations within the general conceptual framework of electron emission phenomenology of metals employing the above "incoming" finalstate wave-function approach. The objective has been to establish the viability of using secondaryelectron emission as a spectroscopic technique for investigating those energy bands at energies above the vacuum level of the solid which are not normally accessible directly by other electron spectroscopies. The results of this work on tungsten show that the energy resolution and $\vec{K}$-space selectivity of the theoretical and experimental spectra have reached a level where SEE measurements now make this goal possible. Also, the technique allows us to study the characteristics of the electron emission process, over a wide range of energy and $\overrightarrow{\mathrm{K}}$ space, free of those complications such as are inherent in the photoexcitation matrix element in photoemission ${ }^{21}$ or in the shape of the tunneling barrier in field emission. ${ }^{23}$ This being so, the SEE spectral fine structure reflects directly the intrinsic electronic properties of the semi-infinite solid, i.e., the surface as well as the bulk crystal.

Following a description of the experimental approach, Sec. II, the angle-dependent measurements, $j_{\mathrm{SEE}}(E, \theta)$ and their phenomenological interpretation, are described in Sec. III. Secondaryelectron-emission spectral fine structure is related to the one-dimensional density of bulk states for the specific conditions which determine emis- sion normal to a low-index surface (Sec. III A); umklapp (elastic) scattering contributions and the problem of off-symmetry line analysis is described (Sec. III B); evidence for intrinsic surface contributions to the secondary-electron current is presented (Sec. III C); and lastly, the importance of quantum-mechanical wave matching at the metalvacuum interface is shown for two explicit cases of enhanced emission arising from resonance effects in the transmission probability function (Sec. IIID). Concluding remarks and summary are given in Sec.IV. Those effects which relate to the overall accuracy of the bulk energy-band-structure calculations are detailed in a separate companion theory paper,${ }^{20}$ together with a wave-mechanical formulation of secondary-electron emission in the light of these new results.

\section{EXPERIMENTAL}

Details of the experimental arrangement, described schematically in previous publications,$^{15}$ are shown in Fig. 1. Angle-resolved SEE spectra reported in this paper were obtained using a $130^{\circ}-$ sector cylindrical electrostatic analyzer with an energy resolution of $0.5 \%$ equivalent to an angular resolution of less than $0.1^{\circ}$, as defined by the entrance slit $\left(0.15 \times 5 \mathrm{~mm}^{2}\right)$. The target crystal could be rotated about an axis normal to the plane of the figure (polar angle $\theta$ ) and about an axis normal to the crystal surface (azimuthal angle $\phi$ ). A small retarding-field analyzer could be rotated out of the plane of the figure. The energy resolution (of the order of $2 \%$ ) and acceptance angle $\left(2^{\circ}\right)$ of this latter analyzer was not suited, however, to obtaining the necessary energy resolution of the final-state spectral fine structure. More important, due to its close proximity to the sample, it created electrostatic patch field effects (arising from work-function variations), which seriously distorted the angular distribution of the lower-energy secondary electrons, $E_{\mathrm{kin}} \lesssim 10 \mathrm{eV} .{ }^{24}$ Therefore, this analyzer was rotated out of the LEED optics grids (or completely removed in some cases), and detailed measurements were made using the "screened" $130^{\circ}$ analyzer alone. It was useful however for providing a cross check on the reproducibility of the overall SEE spectral profile and the gross changes which occured with angular variation. The first grid of the LEED optics was grounded in order to provide a hemispherical drift region free from such effects; "free" in the sense that a residual, but uniform, electrostatic field could be balanced out by applying a small negative potential, $V_{s} \simeq-0.75 \mathrm{~V}$, to the specimen crystal. Ambient magnetic fields were reduced below $10 \mathrm{mG}$ by enclosing the scattering and analy- 


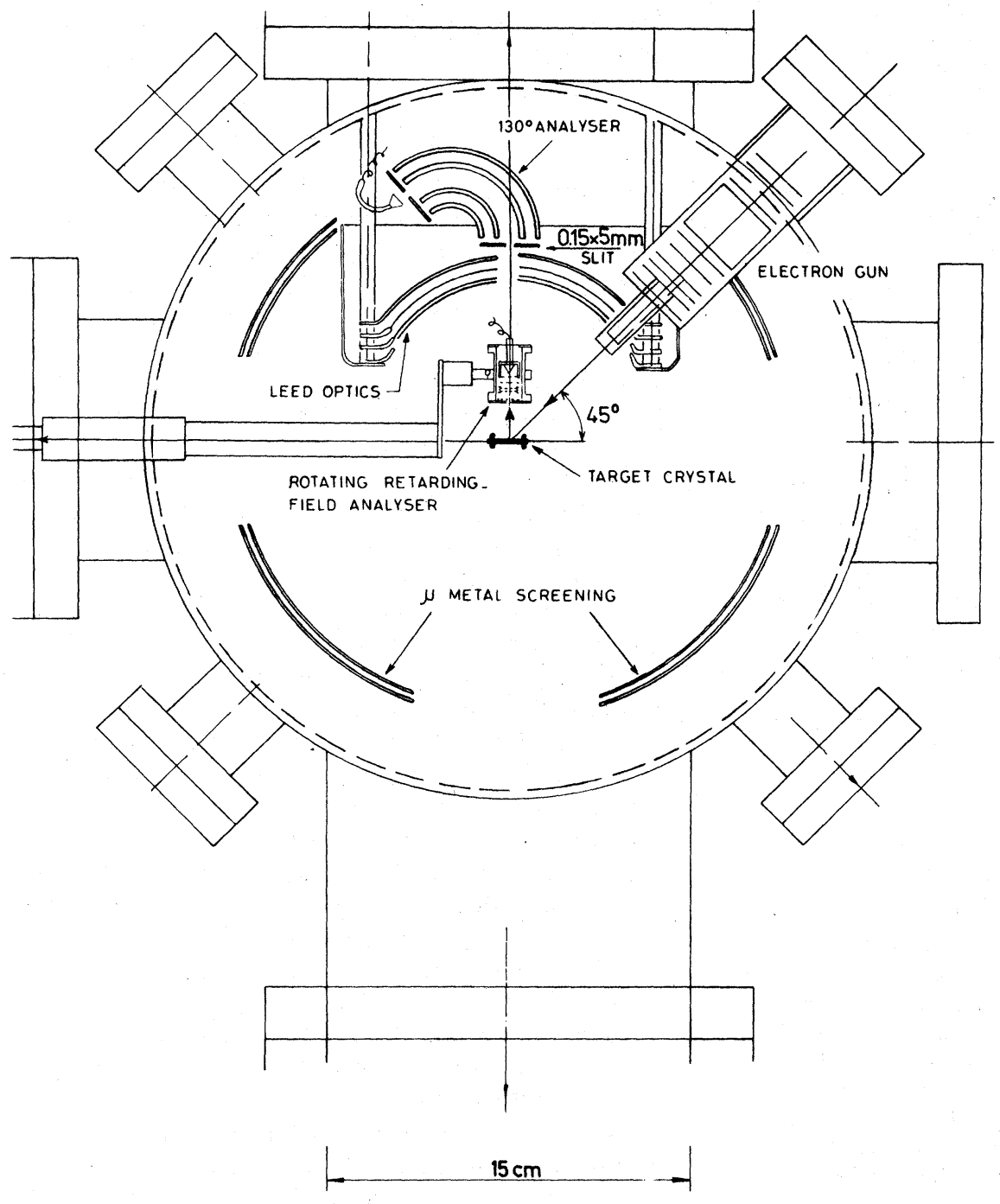

FIG. 1. Angle-resolved SEE experimental highvacuum arrangement showing the relative positions of the primary-electron beam $\left(E_{p} \simeq 100 \mathrm{eV}\right)$ incident at $45^{\circ}$ to the targer crystal normal, the LEED-Auger optics, the angular selective slits, and the $130^{\circ}$-sector cylindrical electrostatic analyzer. The apparatus also contained a small retarding field analyzer, which could be rotated out of the plane of the figure so as to provide a cross check on the gross features of the SEE energydistribution spectra. zing region completely with Mumetal shells.

The samples's surfaces were regularly checked in situ for cleanliness and structure by the LEEDAuger display system contained in a standard vacuum system with a background pressure of better than $1 \times 10^{-10}$ Torr. The LEED-optics systems could also be used for recording large-angle (integrated or random- $k$ ) SEE energy-distribution curves. ${ }^{13,14}$ The electron beam was incident at an angle of $45^{\circ}$ to the crystal surface normal and had a beam-energy spread of around $0.3 \mathrm{eV}$ at the beam energy $E_{p} \simeq 100 \mathrm{eV}$ used in these experiments. The results were not particularly sensitive to primary beam energy, energy spread, or angle of incidence. ${ }^{25}$ Spectrum scanning times were of the order of a few minutes in order to avoid effects due to adsorbate-induced resonances which were manifest even at submonolayer cover- ages. $^{26}$ Care was taken therefore to ensure reproducibility of the recorded spectra. Digital signal storage and analysis was achieved by using a commercial multichannel analyzer and microprocessor computer facilities. Measurements were made on tungsten single-crystal bars $\left(15 \times 5 \times 0.1 \mathrm{~mm}^{3}\right)$, cut to expose the principal low-index (100), (110), and (111) faces. After a standard cleaning procedure ${ }_{9}^{27}$ involving exposure to oxygen at high temperatures to remove interstitial carbon followed by flashing to $2500{ }^{\circ} \mathrm{K}$, the target crystals were oriented to within $1^{\circ}$ using the LEED display system.

\section{RESULTS AND DISCUSSION}

A particular feature of the energy band structure of tungsten is that it exhibits a gap in the unbound 


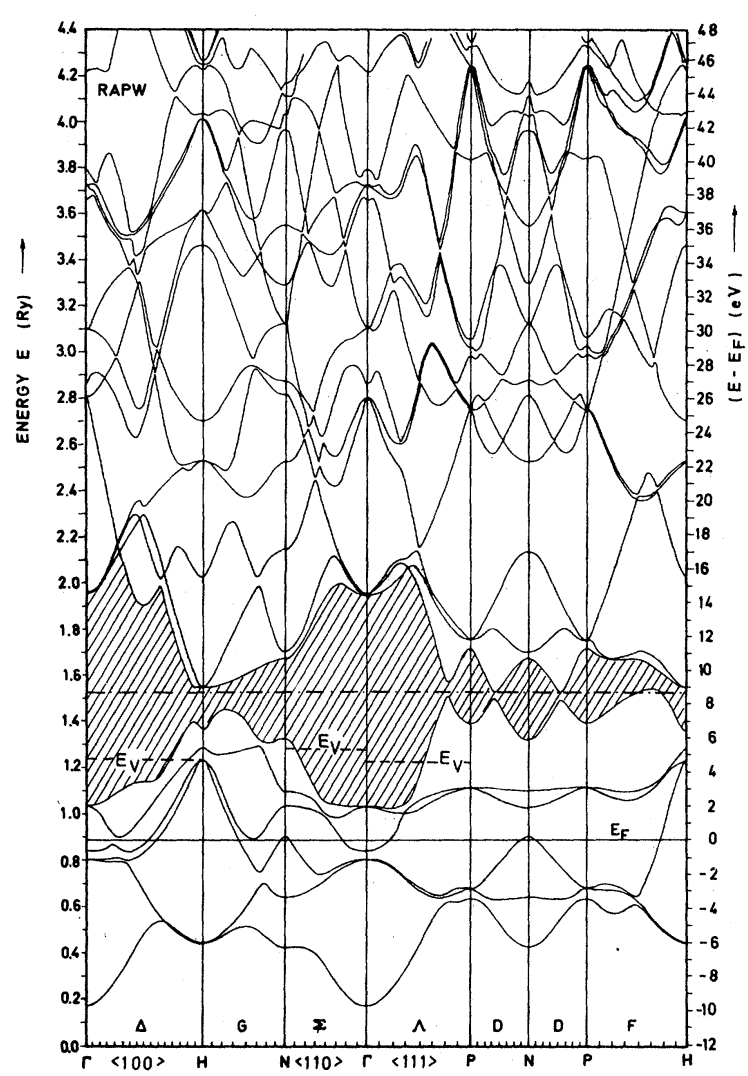

FIG. 2. Electronic energy band structure of tungsten along symmetry directions as calculated and extended to high energies by a relativistic augmented-plane-wave (RAPW) method (Ref. 20). The work functions of each low-index face are indicated by the positions of the respective vacuum levels $E_{v}$ relative to the Fermi level $E_{f}=0 \mathrm{eV}$. A prominent feature is the band gap about the dash-dot line (shaded) which extends throughout the Brillouin zone, except for the region along the $P H$ line (see text).

states bands which extends throughout the Brillouin zone, centered approximately $9 \mathrm{eV}$ above the Fermi energy (dash-dot line in the shaded region, Fig. 2). Previous calculations ${ }^{28}$ have not predicted such an effect. This feature is clearly indicated by a minimum in the experimental SEE spectra, the angular dependence of the width showing good agreement with the behavior predicted by the bulk energy-band structure, Fig. 2. More importantly, since this gap extends throughout the zone (except, that is, for a small region along the $P H$ line) it is sharply defined in all the spectra due to the fact that thermal diffuse (phonon) scattering into the gap from the other states spread throughout the zone at this energy is absent. This being the case, its presence allows us to identify any contributions due to umklapp scattering and/or surface emission occurring in this region. We will the refore pay special attention to this feature in what follows.

\section{A. Symmetry-oriented normal SEE}

Preliminary measurements ${ }^{15}$ of angle-resolved energy-distribution spectra of secondary electrons emitted normal to tungsten crystal (100), (110), and (111) faces, which we shall term symmetryoriented-normal emission, are reproduced in Figs. 3(a), 3(b), and 3(c), respectively. Clearly resolved minima, extending from $2.5<E_{\text {kin }}<4.5$ $\mathrm{eV}, 0.8<E_{\mathrm{kin}}<5.4 \mathrm{eV}$, and $3.2<E_{\mathrm{kin}}<4.4 \mathrm{eV}$ are indicative of the energetic positions and widths of the above gap along the three principal-symmetry lines $\Gamma H(\Delta\langle 100\rangle), \Gamma N(\Sigma\langle 110\rangle)$, and $\Gamma P(\Lambda\langle 111\rangle)$, as indicated by the superimposed energy bands. Also, other fine structure appearing in the spectra appears to be in agreement with the superimposed calculated one-dimensional density of states $D(E)$ along these directions, ${ }^{17}$ entirely analogous to similar directional photoemission studies conducted earlier. ${ }^{16,19}$ The significance of these results lies in the fact that the integrated density-ofstates curve for the full Brillouin zone ${ }^{19}$ shows no gap whatsoever at these energies, nor does the SEE energy distribution using large-angle hemispherical LEED-optics collectors,${ }^{10}$ thus confirming both the directional aspects of the angle-resolved results, and the overall accuracy of the band calculation (Fig. 2).

Peaks in the calculated $D(E)$ curves (Fig。3) assume a constant Lorentzian full width at half height (FWHH) broadening of $0.3 \mathrm{eV}$. (The shaded peak at $E_{\text {kin }}=0 \mathrm{eV}$ [Fig. 3(a)] appears in all experimental spectra and is spurious in that it arises due to scattering effects in the LEED-optics grids at the entrance to the $130^{\circ}$ analyzer (Fig. 1)). As indicated in Figs. 3(a)-3(c), the intensity of spectral fine structure is well above the background noise level so that smoothing was not necessary for emission in the normal direction. Also, the spectra were recorded at an analyzer pass energy of $50 \mathrm{~V}$, equivalent to an energy resolution of $0.25 \mathrm{eV}$. No noticeable sharpening-up of the spectral features was observed at lower pass energies (i.e., energy resolution $<0.25 \mathrm{eV}$ ). It can be seen that the widths of peaks in the SEE spectral fine structure appear to increase with increasing energy, a rather sudden smearing-out of the fine-scale features occurring for energies, $E_{\mathrm{kin}} \geq 20 \mathrm{eV}$. In the $\mathrm{W}(100)$ case, Fig. 3(a), well-resolved maxima centered around 2.5, 5.0, and $11 \mathrm{eV}$ have FWHH of approximately $0.5,1.0$, and $3 \mathrm{eV}$, respectively. The sharp peak at $3 \mathrm{eV}$ in the $\mathrm{W}(111)$ spectrum, Fig. 3(c), has a FWHH of the order of $0.75 \mathrm{eV}$. Such fine-scale structure appears to be absent in 

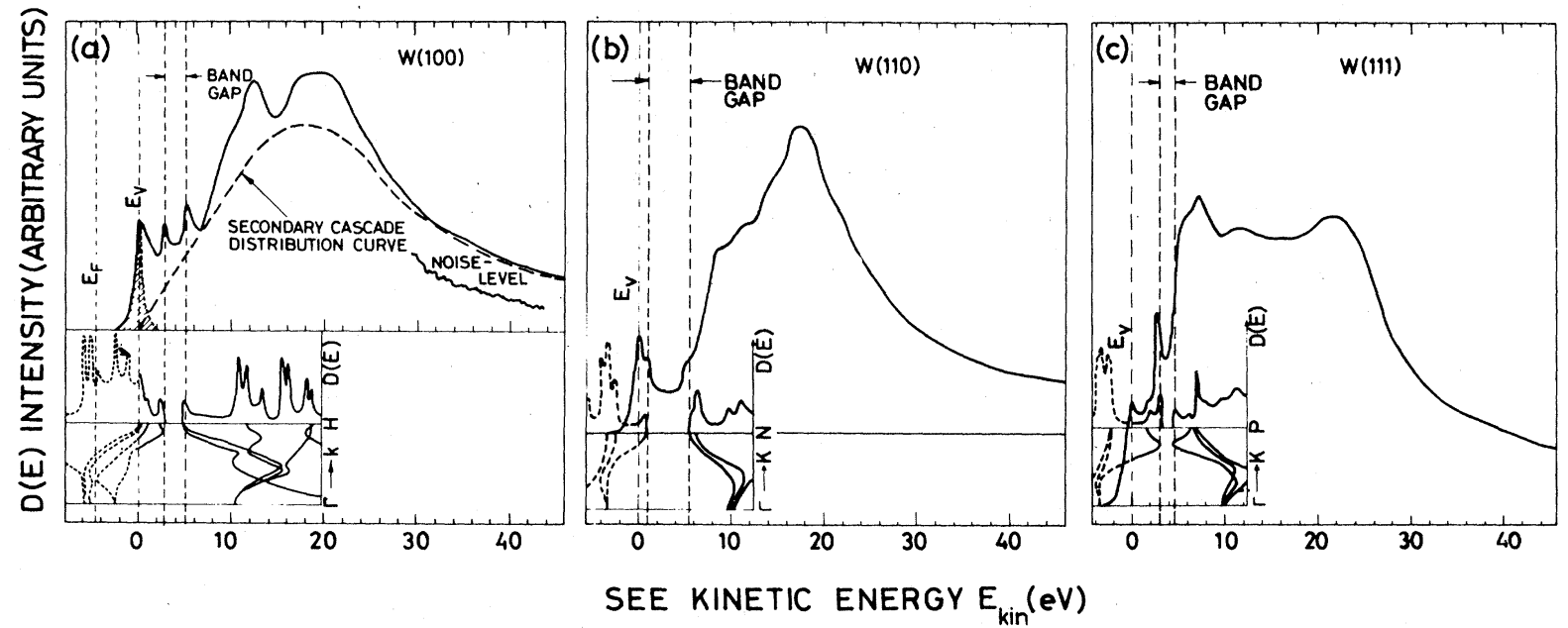

FIG. 3. Symmetry-oriented directional secondary-electron emission normal to (a) W(100), (b) W(110), and (c) W(111) surfaces. The one-dimensional bulk bands and density of states $D(E)$ along the associated symmetry lines are shown for comparison. The SEE kinetic energy $E_{\text {kin }}(\mathrm{eV})$ is referred to the vacuum level $E_{v}$. The positions of energy band-gap features are indicated. The crosshatched peak at $E_{\mathrm{kin}}=0 \mathrm{eV}(\mathrm{a})$ is an experimental artifact which appears in all spectra.

the case of the $\mathrm{W}(110)$ spectrum, except perhaps near the band-gap extremeties. Another feature of importance in all three spectra (Fig. 3 ) is the intensity beneath the curves in the band-gap regions, i.e., the emission does not fall to zero. In order to make a more detailed comparison between the experimental and theoretical density of bulk states profiles, it is clearly desirable to eliminate the background "cascade" curve [Fig. $3(\mathrm{a})$ ]. The problem, however, is in the choice of an appropriate cascade distribution function since this will seriously "weight" the relative intensities of the predicted $D(E)$ fine structure. An obvious first step might be to take the ratio of the actual energy distribution to the free-electron energy distribution $j_{\mathrm{SEE}}(E, \Omega) / j_{\mathrm{SEE}}^{\mathrm{FE}}(E, \Omega)$ analogous to the procedure employed to enhance field-emission energy-distribution fine-scale effects. ${ }^{29}$ Quantitative SEE calculations to date $\mathrm{e}^{5-9,30}$ have employed a free-electron-gas model. These model calculations predict a spectrum of the form

$$
N_{\mathrm{SEE}}\left(E_{\mathrm{kin}}\right) \propto \frac{1}{[\mu(E)]^{2}\left(E_{\mathrm{kin}}+E_{0}\right)^{x}},
$$

where $\mu(E)$ is a geometric function related to electron refraction and escape at the surface, corrected for the case of secondary electrons emitted in the normal direction into a small solid angle..$^{11}$ $E_{0}$ is a parameter whose value depends on the theory chosen, and determines the position of the cascade peak maximum $E_{\mathrm{max}}=E_{0} / X, X \simeq 2$. Wolff's analysis $^{6}$ fixes $E_{0}$ to be the inner potential $V_{0}$, whereas Van der Ziel ${ }^{5}$ used an inner potential $V_{0}$ plus a correction term $h^{2} \lambda^{2} / 2 m$, where $\lambda$ is a screening parameter describing the initial screened-Coulomb interaction between a fast energetic primary electron and the lattice electrons. More recent work by Chung and Everhart ${ }^{30}$ predicts $E_{\max }=\frac{1}{3} \Phi_{s}$, where the sample work function $\Phi_{s}$ is the determinant parameter.

This problem of referencing the energies of the secondary electrons therefore places the cascade peak maximum $E_{\max }$ [Fig. 3(a)] at various energies, $\frac{1}{3} \Phi_{s} \simeq 1.5 \mathrm{eV}$ or $\frac{1}{2} V_{0} \simeq 10 \mathrm{eV}$, taking $\Phi_{s} \simeq 4.5$ $\mathrm{eV}$ and the inner potential $V_{0} \simeq 20 \mathrm{eV}$ from LEED intensity measurements on tungsten. ${ }^{31}$ A parameterized fit to the experimental data of Seah ${ }^{11}$ gave the relation $\left(E_{\mathrm{kin}}+4.5\right)^{-x}$, where $x=2.0$ for SEE from silver and 1.6 from copper. The present SEE results [curves $C$, column (a), Fig. 4], however, would appear to place $E_{\max } \simeq 10$ to $20 \mathrm{eV}$, endorsing a cascade spectrum of the form predicted by the theory of Wolff ${ }^{6}$ (with energies referenced to the energy-dependent inner potential of the metal $V_{0}$ ) based on similar unbound-state wave functions as those relevant to LEED. The schematic curves C, Fig. 4, indicate, however, that no single cascade distribution curve serves to fit the angle-dependent spectra (SE) from all three faces. The "cascade" curves are significantly different in shape for all three crystal orientations that it is arguable as to what constitutes a best-fit. For example, the (110) curve $C$ [column (a), Fig.4] actually appears to give negative enhancement of the experimental spectrum for $E_{\mathrm{kin}} \lesssim 10 \mathrm{eV}$.

In view of the inconsistences inherent in the determination of the cascade distribution curves $C$ described above (and the absence of any complete 


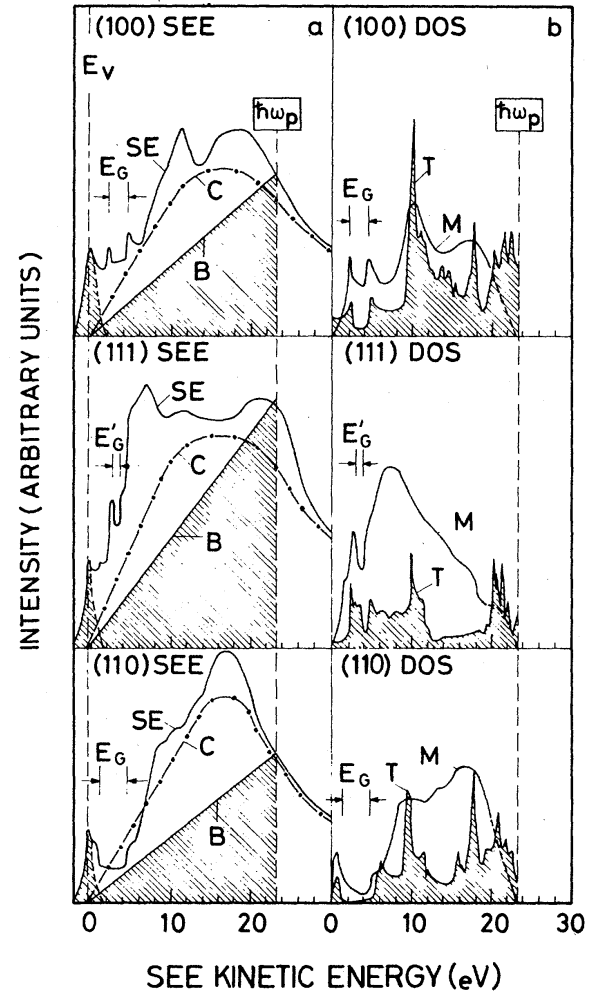

FIG. 4. Angle-resolved energy distribution spectra of secondary electrons emitted normal $\left(\theta \simeq 0^{\circ}\right)$ to the low index (100), (111), and (110) faces of tungsten [curves SE, part (a)]. The SEE cascade distribution curve $C$ is shown schematically. The measured or enhanced density-ofstates (DOS) profiles [curves $M$, part (b)], obtained by subtracting the background $B$ [shaded, part (a)] from the SE spectra, are compared with the theoretical DOS profiles [curves $T$, part (b)], determined for $k$ vectors confined to the first Brillouin zone.

SEE theory), as a first step we have taken the worst-fit approach of simply subtracting an arbitrary linear background, $B$ (shaded region, lefthand column, Fig, 4), which extends from the vacuum level $E_{\mathrm{kin}}=0 \mathrm{eV}$ to approximately the bulk plasmon threshold $\hbar \omega_{p} \simeq 24.0 \mathrm{eV}$. (This particular value has no special significance other than approximating the calculated free-electron value of $22.8 \mathrm{eV}$ and the mean values of the principal surface and volume plasmon losses observed in tungsten, which cover a range of values $\hbar \omega_{p}=20-25 \mathrm{eV}$ reported in the literature..$^{10,32,33}$ ) Since our immediate purpose is to compare the theoretical onedimensional single-particle density of states (DOS) with the measured SEE fine structure, this approximation suffices for the present, as revealed by the measured $(M)$ and theoretical $(T)$ DOS profiles [right-hand column, Fig. 4(b)].34 The curves ( $M$ and $T$ ) show good agreement particularly for energies $E_{\mathrm{kin}} \lessgtr 20 \mathrm{eV}$, in the case of the (100) and
(110) faces. The energetic positions of the finalstate band gaps $E_{g}$ referred to in previously published work, ${ }^{15}$ are clearly resolved as spectral intensity minima in agreement with the theoretically predicted widths. Also, the envelope of the broadened structure occurring at higher energies continues to show reasonably good agreement with that predicted by the bulk contributing states alone, considering the fact that the arbitrarily chosen background (curve $B$ ) will distort spectrum $M$, and severely so for energies above $E_{\mathrm{kin}} \simeq 20 \mathrm{eV}$ (as indicated by the dashed continuation of the $M$ curves in this region). Attention is drawn to the fact that the agreement is not so good in the case of $\mathrm{W}(111)$. This is a point of some importance in the subsequent analysis. At this stage, we merely point out that the band gap predicted along the $\Gamma P$ symmetry direction [Fig。3(c)] and the corresponding minimum in the one-dimensional DOS [Fig. 4(b)] correlates with a minimum in the W(111) normal emission spectrum.

\section{B. Angle-dependent SEE measurements}

Angular anisotropies in SEE arise from conditions imposed at the surface of conservation of parallel momentum and selective wave matching between the crystal wave functions and the planewave components outside the crystal.

Considering a wave packet propagating inside a crystal in a general direction $\vec{k}$, its wave function may be expanded in the form

$$
\psi(\overrightarrow{\mathrm{k}})=\sum_{\vec{G}} C_{\overrightarrow{\mathrm{G}}}(\overrightarrow{\mathrm{k}})|\overrightarrow{\mathrm{k}}+\overrightarrow{\mathrm{G}}\rangle,
$$

the summation extending over all reciprocal-bulklattice vectors $\vec{G}$. The experimental arrangement used previously, ${ }^{15}$ viz., observation normal to a low-index symmetry plane (Figs。 3 and 4 ), has a specific advantage as regards the contributions of higher-G components ("umklapp contributions") to the observed spectra. As pointed out in an earlier paper, ${ }^{35}$ this is best visualized in the extendedzone scheme [Fig。5(a)] which shows an appropriate section through the reciprocal lattice for a bcc crystal, containing the $\langle 100\rangle,\langle 110\rangle$, and $\langle 111\rangle$ directions. The corresponding symmetry lines in the first Brillouin zone [Fig. 5(b)] are included for reference purposes. The vector $\vec{A}$ indicates a general direction in reciprocal space. The various plane-wave components $|\overrightarrow{\mathrm{k}}+\overrightarrow{\mathrm{G}}\rangle$ of a Bloch wave propagating in this direction can be.regarded as deriving from the sections of $\bar{A}$ passing through the Brillouin zones corresponding to reciprocallattice vectors $\vec{G}$. These sections may be projected back into the first zone, as indicated for the $\langle 110\rangle$ direction in Fig. 5(a), so that for a general 

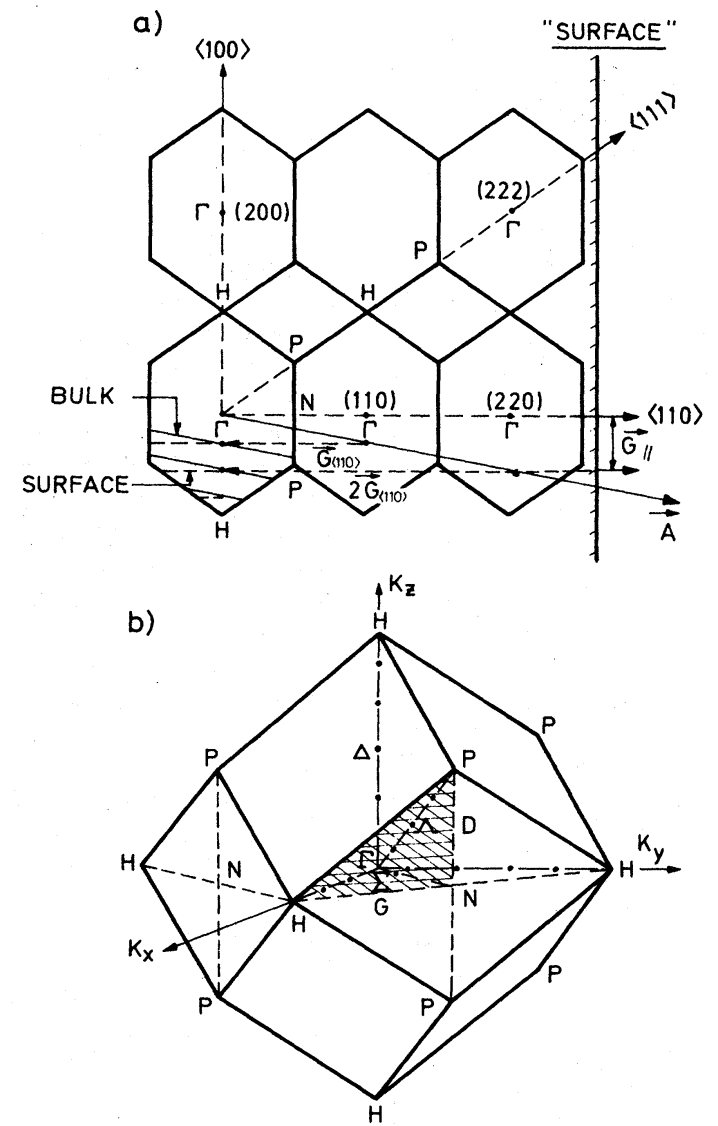

FIG. 5. (a) Section through the reciprocal lattice for a bcc crystal showing the $\langle 100\rangle(\Gamma H),\langle 110\rangle(\Gamma N)$, and $\langle 111\rangle[(\Gamma P)+(P H)]$ symmetry directions in the extended zone scheme. The vector $\vec{A}$ indicates an arbitrary direction in reciprocal space, which can be regarded as being the sum of various components $(\vec{k}+\vec{G})$ projected back into the first zone (lines). Note that the $\left.\vec{G}_{\langle 111}\right\rangle$ vector is not contained in the first Brillouin zone, being the sum of both $\Gamma P$ and $P H$ contributions (see text). The projection of the reciprocal-lattice vectors onto a "surface" plane $\vec{G}_{11}$ is indicated schematically for the (110) face. $\overrightarrow{\mathrm{K}}_{11}$ conservation at the surface produces cylindrical surfaces which sample states throughout the first zone (dashed lines). (b) The first Brillouin zone for bcc tungsten containing the principal symmetry elements, and showing the $\frac{1}{48}$ irreducible zone (crosshatched).

direction $\overrightarrow{\mathrm{A}}$, states located throughout the reduced zone now contribute to emission in this particular direction. The strength of each umklapp contribution $\overrightarrow{\mathrm{G}}_{\langle k k l\rangle}$ is weighted, however, depending on the "strengths" of the various Fourier coefficients $C_{\vec{G}(h k l)}$ in Eq. (2). If, however, $\vec{A}$ corresponds to a symmetry direction such as $\langle 100\rangle$ or $\langle 110\rangle$, all such umklapp contributions project back onto a single line (in this case, $\Gamma H$ and $\Gamma N$, respectively) in the first zone. For this reason, emission from states in these directions may be discussed in terms of the one-dimensional DOS $D(E)$ calculated directly from the energy bands appearing along $\Gamma H$ and $\Gamma N$ in the reduced-zone scheme, $\mathrm{Fig} .2{ }_{0}{ }^{17}$

A problem arises with emission normal to the (111) surface, however, due to the fact that the $P H$ direction is collinear with the $\Gamma P$ principal symmetry line, Fig. 5(a). Electrons emitted along the $\langle 111\rangle$ direction are expected, therefore, to contain contributions from states along $P H$ as well as $\Gamma P$. The significance of this fact is that final states along $P H$ occur with energies which span the band gap in the $\Gamma P$ direction (cf. Fig. 2), but the latter is apparently experimentally resolved at $3.2<E_{k}<4.4 \mathrm{eV}$ [ $E_{G}^{\prime} \mathrm{W}(111)$ (Fig. 4)]. The fact that a gap is resolved in the experimental spectrum for emission normal to $\mathrm{W}(111)$ indicated, in previously published work, ${ }^{35}$ that scattering from the $\mathrm{PH}$ states was of second-order intensity, for reasons which could not be clearly defined at that time. The reason relates to the necessity for wave matching the amplitudes of the various contributing bulk Bloch waves at the surface. This is particularly important for the (111) face, as indicated by the relatively poorer agreement between the (111) one-dimensional $D(E)$ calculated $(T)$ and experimental $(M)$ profiles [Fig. 4(b)] compared with the other faces.

We must recognize also that the SEE current is being emitted from a semi-infinite plane, the twodimensional translational periodicity of which implies that since $\overrightarrow{\mathrm{K}}_{\perp}$ is no longer conserved, the energy eigenstates of the system are characterized by a two-dimensional wave vector $\overrightarrow{\mathrm{K}}_{11}$. Since we are concerned for the moment with that part of the current produced by the bulk Bloch states, we are primarily dealing with the two-dimensional components, $\overrightarrow{\mathrm{G}}_{\|}$, representing the projection of the reciprocal-lattice vectors onto the surface plane, as indicated schematically for the case of emission from a bcc (110) plane [Fig. 5(a)]. The projection of the reciprocal-lattice $\overrightarrow{\mathrm{G}}$ vectors onto this surface is equivalent to erecting a series of right cylinders which project back into the first zone, as indicated in the figure. For the general direction $\vec{A}$, contributions throughout the first zone corresponding to the surfaces of these cylinders contribute. The important point to understand is that it is not the positions of the bands as they appear in the reduced zone scheme, Fig. 2 , that necessarily counts, but rather it is the actual extended $k$-space "momentum content" of the wave functions themselves. Going from a free-electron-like to a "real" solid with a strong scattering pseudopotential produces configuration mixing of the original plane-wave one-electron states so that the eigenfunctions become linear combinations of the (orthogonalized) plane waves. The extent of the ad- 

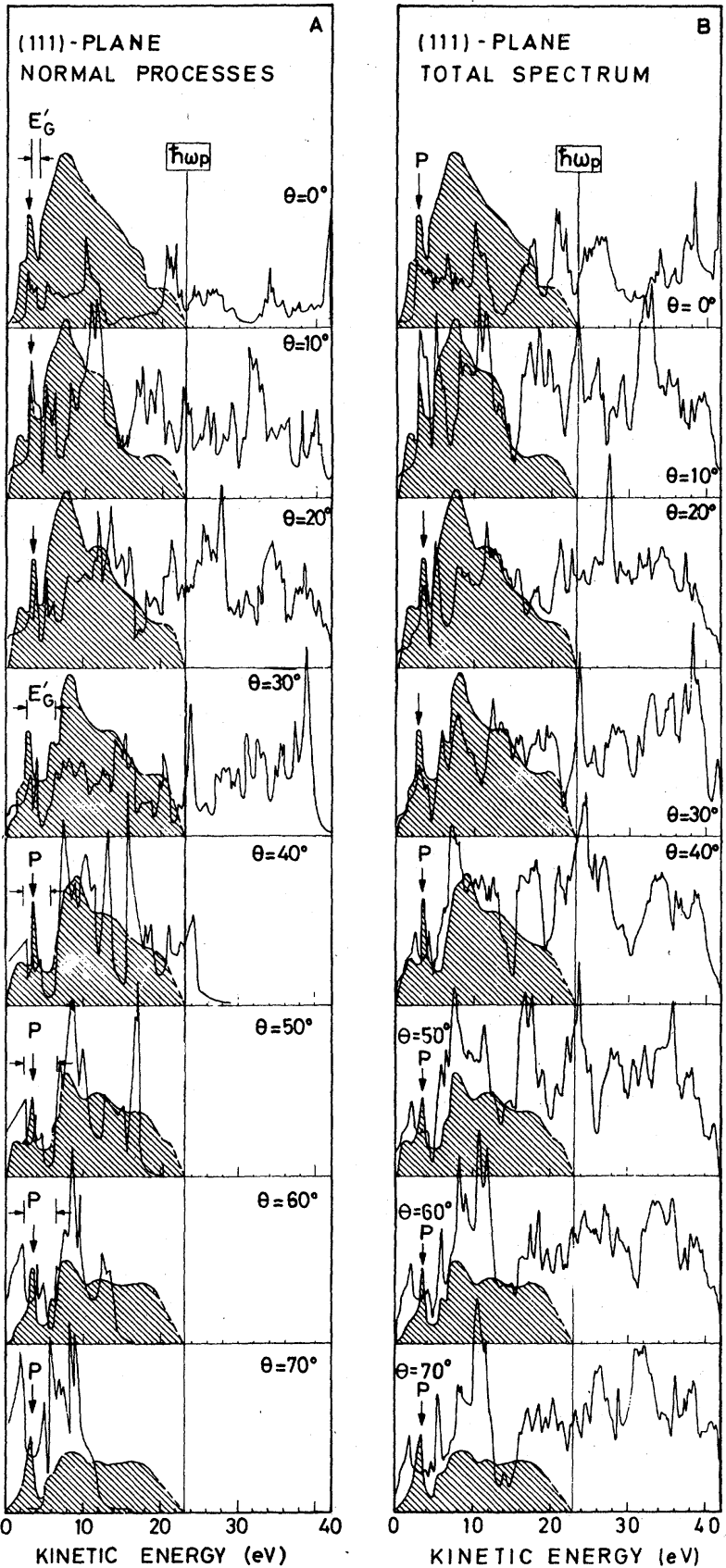

mixture is governed by the pseudopotential chosen as well as by general symmetry considerations . Wave-matching selectivity at the surface will serve to "weight" the various contributing finalstate Bloch wave components contributing to the angle-resolved current $j_{\mathrm{SEE}}(E, \theta)$. For general off-symmetry line directions and $\vec{k}_{11} \neq 0$ this mixing will be even more pronounced and the "weights" of the various plane-wave components will change markedly.

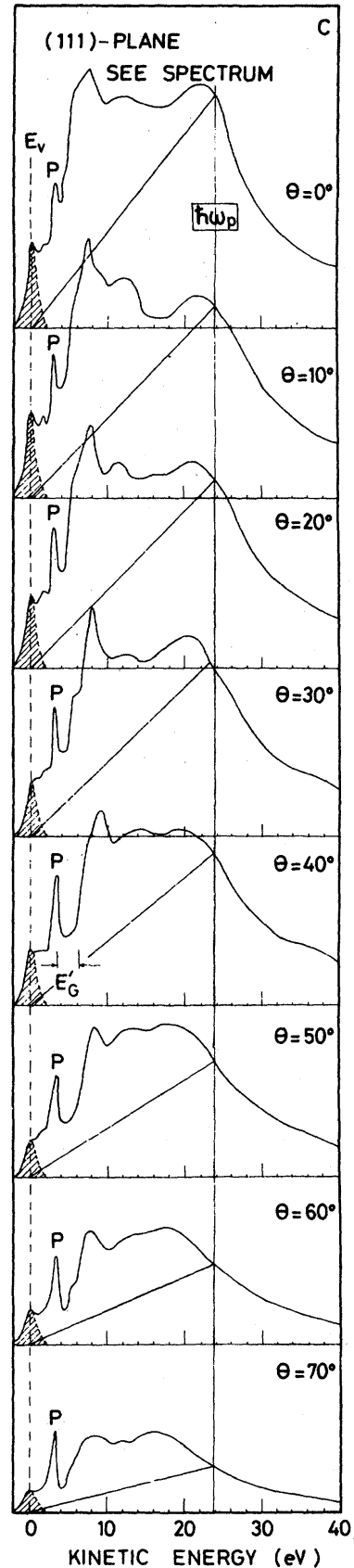

FIG. 6. Angle-dependent SEE spectra for W(111) showing: column (c), the variation of the total spectral intensity with polar angle $0^{\circ} \lesssim \theta \lesssim 70^{\circ}$ in the azimuthal plane containing the $P H$ zone edge line [Fig. 5(b)]; column (b), a comparison of the "enhanced" SEE DOS profiles, $M$ (shaded, cf. Fig。 4) with theoretical "total" bulk DOS profiles including umklapp contributions from directions in the extended zone defined by up to seven shells of low-index $\vec{G}_{\langle h k l\rangle}$ vectors (Ref. 20); and column (a), a similar comparison with theoretical DOS profiles integrated over the reducedzone $k$ space only ("normal" processes). The umklapp contributions [column (b)] were equally "weighted" in intensity since wave matching at the vacuum-crystal interface was not included in the calculations. The overall decrease in SEE spectral intensity with increasing $\theta$ [column (c)] is due to the $\cos ^{2} \theta$ isotropic emission dependence. The "anomalous" peak- $P$ feature and the dimensions of the band gap $E_{G}^{\cdot}$ are indicated.
The point being made is that angle-resolved measurements in off-symmetry directions contain contributions to each element of spectral structure from quite general points throughout $k$ space, i.e., it is rarely the case that any single feature in the spectrum can be identified simply by inspection of the reduced-zone band structure. This implies that although the experiment might refer to a specific direction of emitted current, detailed calculations relating to extended $k$ space are nec- 

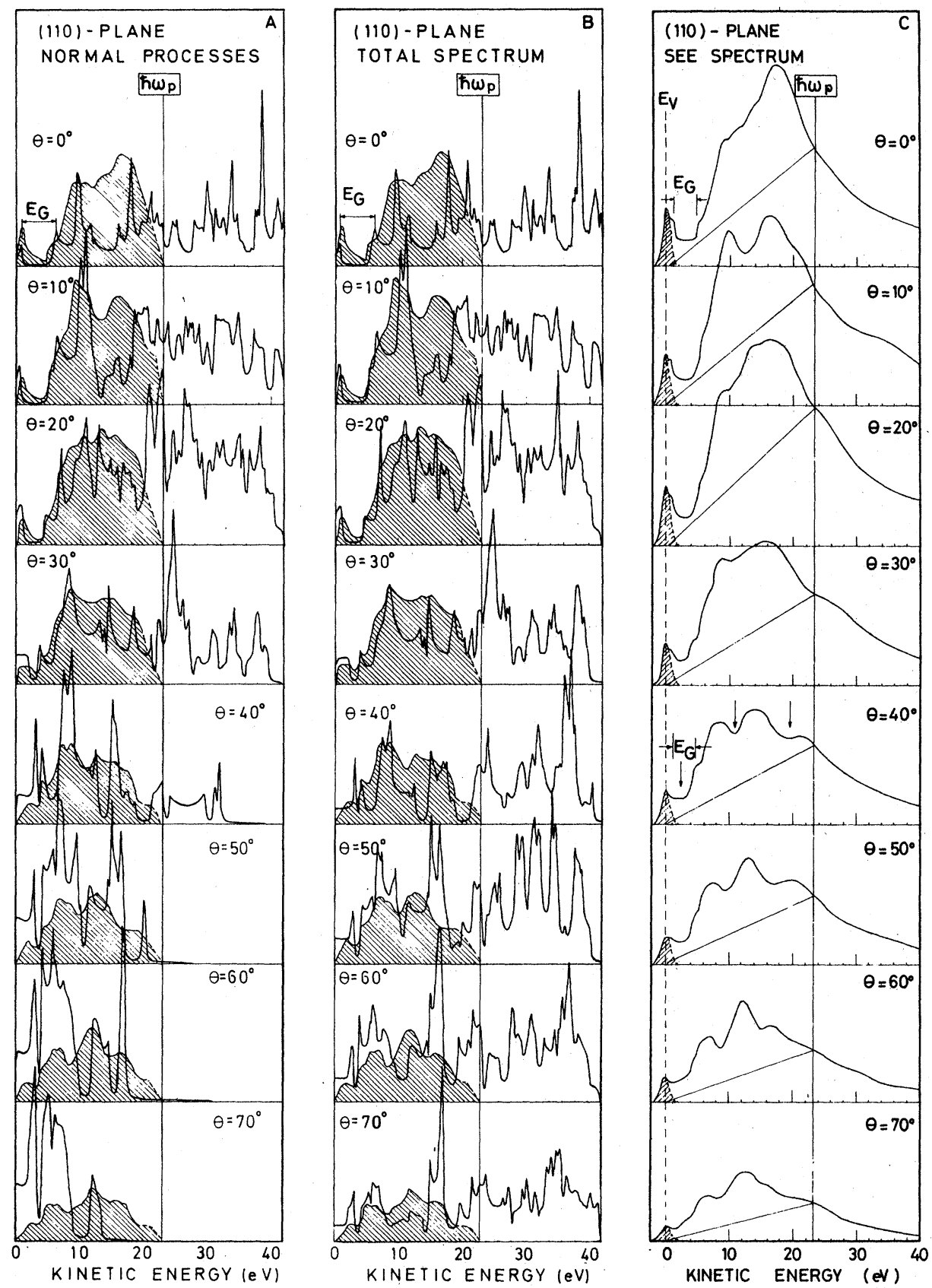

FIG. 7. Angle-dependent DOS profiles for W(110) showing the variation of spectral intensity with polar angle $0^{\circ} \varsigma \theta \leqslant 70^{\circ}$ in the azimuthal plane $\Gamma N \rightarrow \Gamma P \rightarrow \Gamma H$ along direction $D$ [Fig. 5 (b)]. Note the fact that band gaps appear (arrowed) at higher energies than the principal gap $E_{G}$, for large angles $\theta \gtrsim 40^{\circ}$ (see text).

essary to avoid erroneous interpretation in terms of the bulk band structure.

To asses the relative contributions of these umklapp $\vec{G}$ processes, the angular variations of the SEE spectra $\left(0^{\circ} \leqslant \theta \leqslant 70^{\circ}\right)$, shown in parts (c) of Figs. 6-8 for the (111), (110), and (100) faces, respectively, are compared with theoretical curves determined by (a) integrating over the reduced-zone states [normal processes, parts (a)] with (b) theoretical curves which include contribu-

tions from up to seven shells of $\vec{G}$ vectors emanating in all directions about $\Gamma$ in the extended-zone scheme [total spectrum, parts (b)]. ${ }^{20}$ The theoretical distributions are shown superimposed on the "enhanced" SEE spectral profiles extending up to the bulk plasmon threshold (shaded, Figs. 6-8). The theoretical profiles (cf. $T, F i g .4$ ) were determined for specific detector positions relative to the surface normal as follows.

(a) Specify the work functions for each surface: 

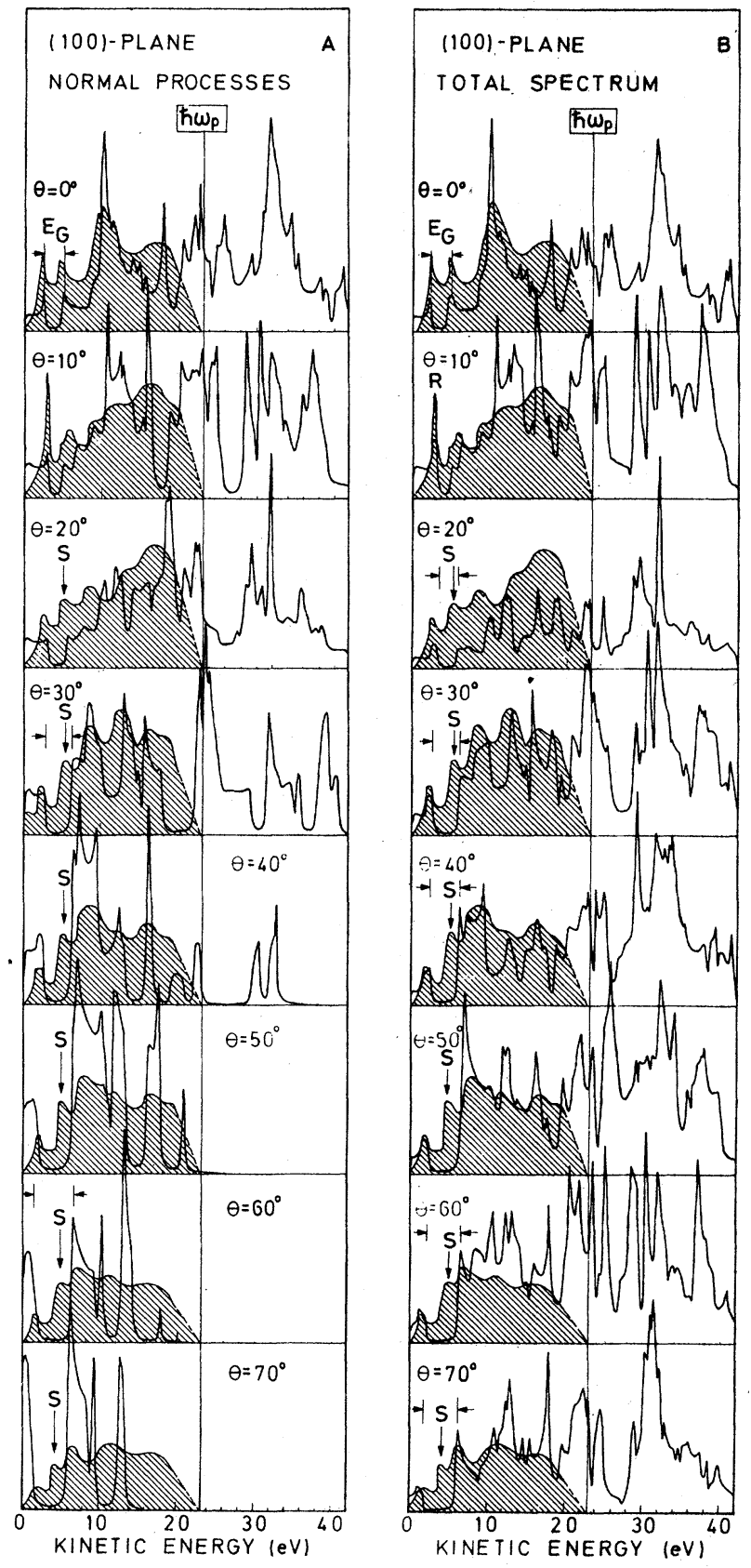

$\Phi_{s}=4.3 \mathrm{eV}$ for (111) and (100) and $\Phi_{s}=5.1 \mathrm{eV}$ for (110); the values being based on previous photoemission work. ${ }^{16}$

(b) Choose detector position, i.e., the external angles $\theta$ and $\phi$. The polar angle $\theta$ is measured from the surface normal and the azimuthal angle $\phi$ measured from some reference line lying in a plane parallel to the surface. Referring to Fig. $5(\mathrm{~b}), \phi$ was chosen such that $\theta$ varied from $\Gamma P$ along the zone-edge line $P H$ over a $70^{\circ}$ range for

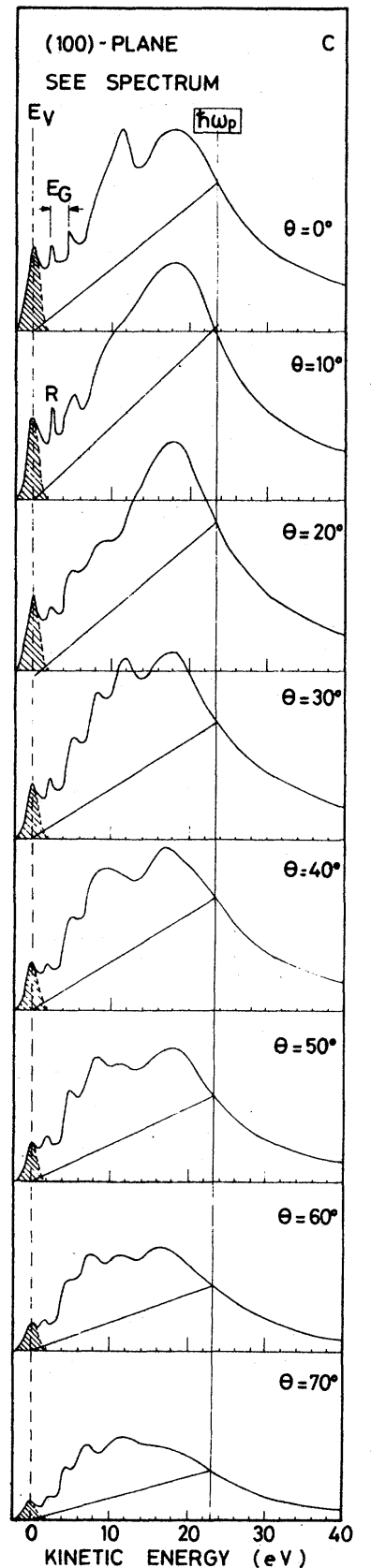

FIG. 8. Angle-dependent DOS profiles for $\mathrm{W}(100)$ showing the variation of spectral intensity with polar angle $0^{\circ} \lesssim \theta \lesssim 70^{\circ}$ in the azimuthal plane $\Gamma H \rightarrow \Gamma N \rightarrow \Gamma H$ along direction $G$ [Fig. $5(b)]$. The "anomalous" peak- $S$ and peak- $R$ features, appearing for angles $10^{\circ} \lessgtr \theta \lessgtr 70^{\circ}$ are discussed in the text. the (111) face, Fig. 6 ; from $\Gamma N$ to $\Gamma P$ to $\Gamma H$ along $D$ for the (110) face, Fig. 7, and from $\Gamma H$ to $\Gamma N$ to $\Gamma H$ along $G$ for the (100) face, Fig。 8 . The measurements thereby scanned the irreducible $\frac{1}{48}$ zone [shaded volume, Fig. 5(b)].

(c) Calculate the number of electrons detectedas a function of kinetic energy $E_{\text {kin }}$ normalizing the distribution arbitrarily to that of the enhanced experimental SEE profiles $M$ [cf. Fig。4(b)].

Umklapp contributions to the spectra are seen 
most clearly by comparing the normal and total theoretical profiles [columns (a) and (b), respectively] with the enhanced SEE spectra (superimposed and shaded in Figs 。6-8). The theoretical results ${ }^{20}$ show quite clearly that, for increasing angles up to $\theta=70^{\circ}$, the overall contribution from umklapp processes increases with increasing $\theta$, and simultaneously contribute to the intensity in the lower-energy regime $E_{\text {kin }} \leqslant 10 \mathrm{eV}$, where the spectral fine structure is most clearly resolved (Figs. 3 and 4). ${ }^{36}$ The SEE spectra resolve additional structure in the region $E_{\mathrm{kin}} \leqslant 20 \mathrm{eV}$ which is absent in the "normal processes" profiles for all three faces. Also, as the detailed comparison between the theoretical and experimental bulk contributing states confirms,$^{20}$ it can be seen that the apparent increased spectral broadening with increasing kinetic energy referred to earlier (Sec. IIIA), is in fact mainly due to an increasing number of energetically closely spaced states contributing to the spectral profiles for energies below the bulk plasmon threshold $\hbar \omega_{p} \simeq 24 \mathrm{eV}$.

The experimental and theoretical bulk singleparticle states spectral profiles, Figs.6-8, show good overall agreement, the band gaps $E_{G}$ referred to above being a particularly prominent feature in the spectra. For example, the angle-resolved SEE measurements on the (111) face [Fig。6(c)], clearly show the gap at $3.2<E_{k}<4.4 \mathrm{eV}\left(E_{G}^{\prime}\right)$ opening up as $\theta$ increases along $P H$ towards the $\Gamma N$ direction [cf。Fig. 5(b)]。 In contrast, the SEE measurements on the (110) face (Fig。7) show the wide band gap $0.8<E_{\mathrm{kin}}<5.4 \mathrm{eV}\left(E_{G}\right)$ along $\Gamma N$ $\langle 110\rangle$, closing with $\theta$ increasing along $D$ in the direction of the $\Gamma P\langle 111\rangle$ projection, as predicted by the theoretical curves [columns (a) and (b)]. The gap along the $\Gamma H\langle 100\rangle$ direction $\left[E_{G}, F i g\right.$. $8(c)$ ] remains a spectral feature of the emission from this face for all polar angles. The (110) spectra [Fig。7(c)] are reflected in the (100) spectra [Fig. 8(c)] as evolving as the detector scans along $G$ from $\Gamma H$ towards the $\Gamma N$ symmetry direction, particularly in the energy range $6 \leqslant E_{\mathrm{kin}} \leqslant 20$ $\mathrm{eV}$ and angles $20^{\circ} \leqslant \theta \leqslant 60^{\circ}$; the origin of the strongly modulated overall spectral envelope intensity is due to additional relative gaps [arrowed, Fig。7(c)] appearing at higher energies, $E_{\mathrm{k} \text { in }}$ $\simeq 10-20 \mathrm{eV}$, which are common to both faces 。

Detailed wave-matching calculations between the unbound-bulk-state Bloch waves carrying the current to the surface and the plane-wave components in vacuum of energy $E_{\mathrm{kin}}=\overrightarrow{\mathrm{p}}^{2} / 2 m$, assuming $(1 / \hbar) \vec{p}=\vec{K}_{\| 1} \equiv \vec{G}_{11}+\vec{k}_{\| 1}$ (where $\vec{G}_{\| 1}$ is the component parallel to the surface of any reciprocal-lattice vector $\vec{G}$ and $\vec{k}_{||}$is the parallel component of the reduced Bloch-wave vector $\vec{k}$ for the electron in the crystal), were not carried out in the calculations ${ }^{20}$
Wave matching is expected to affect mainly the relative intensities of the various states contributing to the fine-scale structure appearing in the theoretical profiles. Wave matching has some important consequences also however, not least in the inclusion of evanescent Bloch functions of complex wave vectors having real $\vec{k}_{\|}$but complex $\vec{k}_{1}$ components, which we will discuss in Sec. III C. The object of presenting the spectral results in the form shown in Figs. 6-8 is to look for a correlation with the bulk densities-of-states profiles in order to distinguish any "anomalous" features arising from (i) intrinsic surface resonances and/ or (ii) the transmission probability $T\left(E, \overrightarrow{\mathrm{K}}_{\|}\right)$。 Three such "anomalies" are apparent in the angleresolved SEE spectra, Figs.6-8. These are (i) the peak labeled $P$ in the W(111) spectra (Fig. 6); (ii) the peak $R$ in the $\theta=10^{\circ}$ spectra for $\mathrm{W}(100)$ (Fig。 8); and (iii) the peak $S$ in the W(100) spectra for $10^{\circ} \lesssim \theta \lesssim 70^{\circ}$ (Fig. 8). These features cannot be explained as being due to umklapp or any bulk density-of-states contributions. ${ }^{20}$ Also, emission from the $W(111)$ surface is particularly anomalous. That is, the apparent gap $\left(E_{G}^{\prime}\right)$ at $3.2<E_{\mathrm{kin}}<4.4 \mathrm{eV}$ $\left(\theta=0^{\circ}\right.$, Fig. 6$)$ is resolved in both the SEE and the normal-processes theoretical profiles but, significantly, is absent in the total density-of-states curve, column (b). The origin of this anomalous gap in the $\mathrm{W}(111)$ symmetry-oriented-normalemission spectrum is clearly related to the origin of the sharp peak $P$, located near the lower edge of the gap in the first Brillouin zone.

These features are indicative of a need to include wave-matching arguments in any analysis of electron emission spectra. As described in a separate theory paper, ${ }^{20}$ however, the computational difficulties are considerable. Nevertheless, it is possible to use qualitative arguments to identify the origin of such effects using the final-state "incoming-wave" concept. Anomalous intensity in the SEE spectral distributions, Figs. 6-8, is shown to originate from two sources (other than the above bulk density-of-states contributions)-surface emission from evanescent unbound states (Sec. III C) and wave-matching conditions which produce a strong enhancement in the transmission probability (transmission resonances) of Bloch waves incident on the surface (Sec.IIID). A full analysis of all the spectral fine-structure contained in the curves (Figs. 6-8) is exceedingly involved and is, therefore, delegated to a companion theory paper ${ }_{0}^{20}$

\section{Surface emission from evanescent unbound states}

The one-electron states in the crystal can be classified according to their reduced momentum (k) which can be decomposed into components par- 
allel and perpendicular to the surface plane under study $\left(\vec{k}_{11}, \vec{k}_{\perp}\right)$ and some band index $n$. The energies of the one-electron states are then defined by some dispersion relation $E_{n}(\vec{k})$. This remains true for Bloch states propagating in the surface as well as the bulk lattice except that the surface states are evanescent wave functions with complex wave vectors having real $\vec{k}_{\|}$but complex $\vec{k}_{1}$ components 。

The eigenstates of the surface can be labeled by a two-dimensional wave vector $\vec{k}_{11}$ which relates to a two-dimensional Brillouin zone associated with the surface crystallography. In the usual way, one can confine the wave vector $\vec{k}_{\| \mid}$associated with these evanescent Bloch functions to lie within a reduced zone, called the surface Brillouin zone (SBZ)。However, since as we have seen in Secs. III A and III B, the secondary-electron current $j_{\mathrm{SEE}}(E, \theta)$ contains a substantial contribution produced by bulk Bloch states, and since these are normally enumerated by using vectors confined to the conventional (three-dimensional) Brillouin zone [Fig.5(b)], it is important to understand the relation between the two types of zones.

Each of the two-dimensional Brillouin zones associated with the principal low-index surfaces of the bcc lattice can be placed into the three-dimensional (first) Brillouin zone in such a way that the borderlines of the surface Brillouin zones fall in the zone faces of the latter, as illustrated in Fig. 9 (shaded areas). The SBZ symmetry elements have been labeled with bars on top to distinguish them from those corresponding to the bulk zone. In some cases, SBZ and bulk zone symmetries are coexistent, which can lead to some confusion. We have the refore chosen to list both where the case demands, following the convention of Koster ${ }^{37}$ in the labeling of the bulk zone symmetries. The irreducible part of the SBZ's is shown crosshatched in each case. One can construct a satisfactory three-dimensional Brillouin zone by using the SBZ as a base and erecting a right cylinder of height $\vec{G}_{0}$, where $\vec{G}_{0}$ is the shortest three-dimensional reciprocal-lattice vector perpendicular to the crystallographic surface under study. The volume of reciprocal space so constructed has all the desired properties but it has only the two-dimensional symmetry of the bulk lattice. The SBZ will have associated group symmetry elements, however, which related to the surface rather than the bulk lattice symmetries.

Considering a particular azimuthal direction $\phi$ in the surface, for each value of $\vec{k}_{11}$ many bulk states project onto the surface plane (since $\vec{k}_{\perp}$ is not conserved), giving rise to broad bands of allowed states. This is shown for the $W(110)$ surface, Fig. 10 (shaded regions), in which the energies of the upper and lower bulk band-gap extrem-

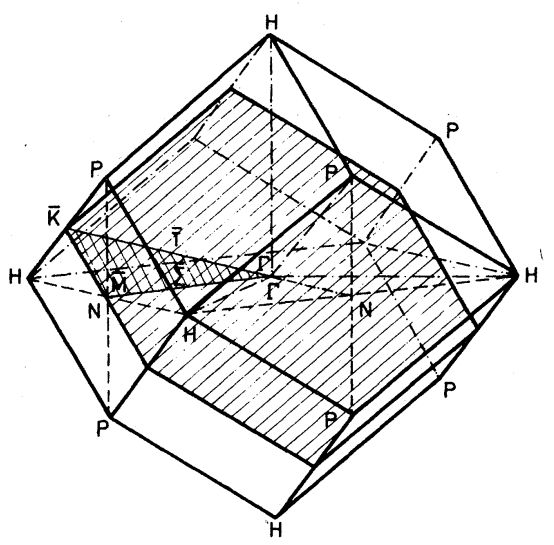

(a) (111)

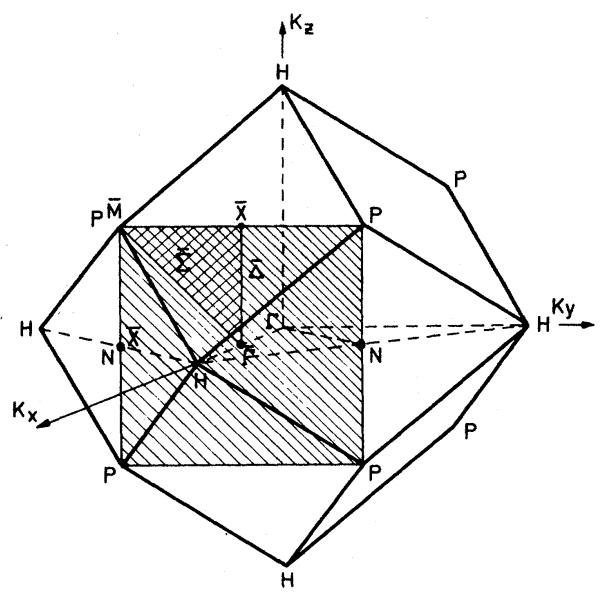

(b) (100)

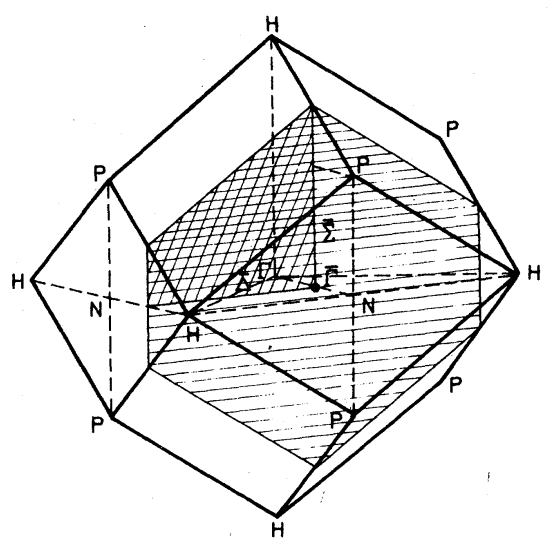

(c) (110)

FIG. 9. Surface Brillouin zones (shaded) for a bodycentered-cubic lattice showing the relationship to the bulk first Brillouin-zone symmetry. The irreducible surface zones are shown (crosshatched), labeled with the two-dimensional symmetry elements (bar on top) for (a) the (111) plane, (b) the (100) plane, and (c) the (110) plane. 


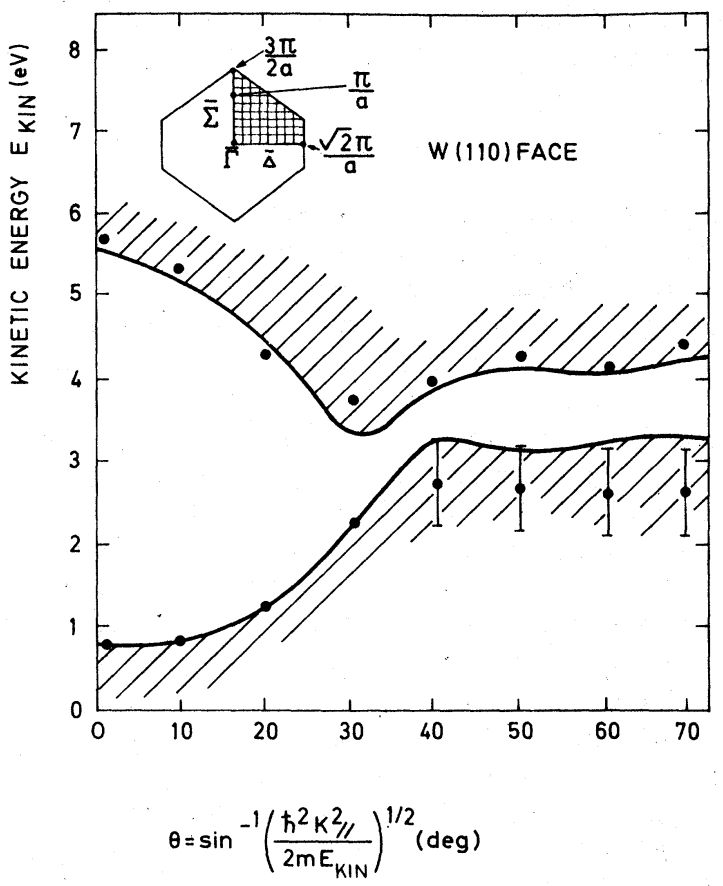

FIG. 10. Angular dependence of the $W(110)$ bulk band gap $E_{G}$ (Fig. 7) as a function of polar angle $\theta=\sin ^{-1}\left[\hbar \overrightarrow{\mathrm{k}}_{i !}^{\prime}\right]$ $\left(2 m E_{\text {kin }}\right)^{1 / 2}$ ]: points (experiment), lines (theory) along the $\bar{\Sigma}$ direction of the surface Brillouin zone (inset). The projection of the bulk zone point $P$ [Fig. 9 (c)] onto the SBZ occurs at $\vec{k}_{\| 1}=\pi / a \approx 1 \AA^{-1}$ as indicated.

ities $\left(E_{G}\right.$, Fig. 7 ) are plotted as a function of $\vec{k}_{\|}$related to the polar angle measurement by the usual free-electron relationship

$$
\theta=\sin ^{-1}\left[\hbar \overrightarrow{\mathrm{K}}_{11} /\left(2 m E_{\mathrm{kin}}\right)^{1 / 2}\right]
$$

along the $\bar{\Sigma}$ symmetry line of the appropriate SBZ, the irreducible part of which is shown crosshatched (inset, Fig. 10). The SBZ boundary in this direction occurs at $\vec{k}_{11}=3 \pi / 2 a \simeq 1.5 \AA^{-1}, a=3.16 \AA$ being the tungsten lattice separation for the (100) cubic face. ${ }^{38}$ The angular SEE measurements (Fig。7) however only extend out to $\pi / a$ or $\vec{k}_{11} \simeq 1.0 \AA^{-1}$, which occurs at $\theta \simeq 70^{\circ}$ (Fig。10). The agreement between the theoretical (curves) and experimental (points) SEE spectral behavior (Fig. 7) is seen to be better than $\pm 0.5 \mathrm{eV}$ over the whole angular range $0 \lesssim \theta \lesssim 70^{\circ}$. This is indicative of the relative sharpness of the spectral resolution of the band edges, particular for $\theta \lesssim 40^{\circ}$, and suggests that the emission at the energetic extremities $E_{G}$ is determined by bulk as opposed to purely surface density of states singularities at these energies ${ }^{29}$ The behavior of the W(111) (Fig。6) and W(100) (Fig. 8) band-gap spectral features serve to endorse this view. However, emission from the (111) and (100) surfaces is complicated by the appearance of

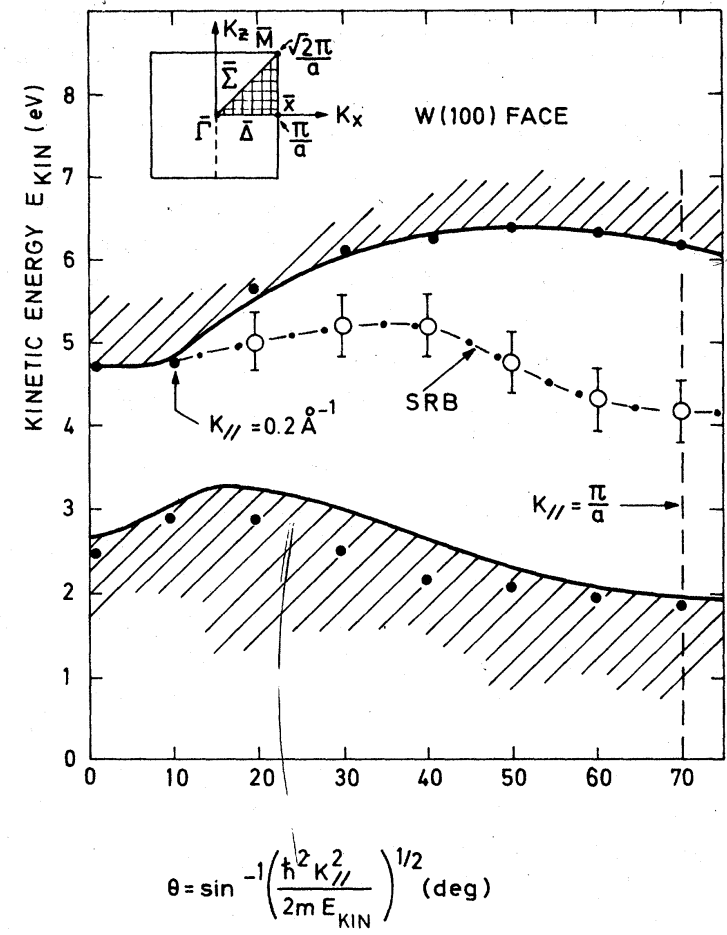

FIG. 11. Angular dependence of the W (100) bulk band gap $E_{G}$ and the SEE spectral peak $S$ (Fig. 8) as a function. of polar angle $\theta=\sin ^{-1}\left[\hbar \overrightarrow{\mathrm{k}}_{|1|} /\left(2 m E_{\mathrm{kin}}\right)^{1 / 2}\right]$ : points (experiment); lines (theory). The surface resonance band (SRB) in this case extends out along the $\bar{\Delta}$ direction to the surface-Brillouin-zone (inset) boundary at $\bar{X}$ [Fig。9(b)], $k_{1 \mid}=\pi / a \simeq 1 \AA^{-1}$ around $\theta \simeq 70^{\circ}$, as indicated.

"anomalous" fine structure which appears within the band-gap regions indicated as $E_{G}\left[i_{0} \Theta .\right.$, peak $P$ (Fig。6) and peaks $S$ (Fig。8), respectively].

\section{Surface resonance bands}

A band of surface states (resonances) ${ }^{39}$ can arise in the energy gap between the bulk states as a consequence of the periodic atomic lattice arrangement in the surface. In Fig.8, all of the SEE spectral fine structure appearing in the $\mathrm{W}(100)$ curves $M$ (shaded) may be explained in terms of bulk contributing states (curves $T)^{20}$ except, that is, for the anomalous SEE spectral intensity, peak $S$, which evolves into the band-gap region $E_{G}$ with increasing polar angle $\theta$ (and the extremely sharp peak $R$, which we will discuss subsequently). This feature $S$ is quite distinct from any possible umklapp effects, as seen by comparing parts (a) and (b), Fig. 8, and identifies with emission due to an intrinsic surface resonance effect. This may be seen by plotting the dispersion behavior of peak $S$ (shown arrowed, Fig. 8), together with the (100) band-gap extremities, as a function of $\theta=\sin ^{-1}\left[\hbar \vec{k}_{\| 1} /\left(2 m E_{\mathrm{ktn}}\right)^{1 / 2}\right]$. The re- 
sults scanning along the $\bar{\Delta}$ SBZ symmetry direction are shown in Fig.11. The point of interest, in this case, is that the behavior of this "surface resonance band" (SRB) is clearly indicative of the SBZ boundary $\bar{X}$ occurring at $\overrightarrow{\mathrm{k}}_{11}=\pi / a \simeq 1 \AA^{-1}$ or $\theta \simeq 70^{\circ}$ (vertical dashed line). Again, the agreement between the theoretical (curves) and experimental (points) is very close for the band-gap edges, as in the case of $\mathrm{W}(110)$, Fig. 10 。

The two-dimensional band structure for the prominent surface states/resonances associated with the clean $\mathrm{W}(100)$ surface, determined by a parametrized linear-combination-of-atomic-orbitals (LCAO) slab-model calculation, ${ }^{40}$ predicts just such a band of unbound one-electron "states" existing above the vacuum level as that experimentally resolved in Figs. 8 and 11.A comparison between the calculations ${ }^{40}$ and the SEE measurements (Fig. 11) show good agreement in the appropriate energy range and with the correct sense of dispersion (downward in energy away from $\bar{\Gamma}$ ) along $\bar{\Gamma} \bar{X}$ or $\bar{\Delta}$ symmetry direction. ${ }^{41}$ The LCAOmodel calculations identify the above SRB with eigenvector components derived from $\mathrm{W} 6 p$ orbitals, the largest component of which changes from an admixture of $P_{z}-6 s$ character close to $\bar{\Gamma}$, the $P_{x}$ component increasing and becoming dominant (as one might expect) at the larger polar angles. ${ }^{42}$

The results to date $e^{40,42}$ would indicate that the surface states (resonances) are a common feature of the W(100) surface. For example, a band of bound surface "states" is known to exist around $-0.4 \mathrm{eV}$ below the Fermi level $E_{F}$, and has been observed to produce strong spectral effects in both field- and photoelectron emission measurements ${ }_{\circ}^{43}$ These states appear to have LCAO eigenvector $\mathrm{W}$ orbital components which are conspicuously large in the outermost atomic layers, the admixture of which in many cases varies with the magnitude of $\vec{k}_{\| 1}$. That is, the origin of the associated surface emission relates to the orbital content of the surface local density-of-states contribution to the emitted current. This would indicate that the emission specifically relates to the energy distribution of the "spectral density of states in the surface" in these circumstances (Feuchtwang and Cutler, Ref. 23).

Similar unbound SRB behavior to that shown in Fig. 11, but produced by ordered monolayers of adsorbed gases, has been resolved in the W(110) band gap (Figs . 7 and 10) under symmetry-oriented normal SEE conditions. ${ }^{26}$ These latter adsorbateinduced resonances disappear when the adsorbate "superlattice" disorders under thermal treatment. These "extrinsic," together with the above "intrinsic," effects provide clear evidence for evanescent Bloch waves propagating in fairly narrow bands extending throughout the respective surface Brillouin zones (Fig. 9).

\section{Surface emission}

For energies above the vacuum level $E_{v}$, the continuum of plane-wave vacuum states are always able to penetrate a short distance into the solid, and represent a finite probability of an electron arriving at the detector from a source of excited atoms located in the outermost layer. For each value of $\vec{k}_{11}$ throughout the surface Brillouin zone, the associated final-state plane-wave function will have a continuous value and normal derivative at every point in the surface unit cell ${ }^{44}$ and so contributes to the total emission $j_{s}(E, \Omega)$. Specifically, this "tailing" of the vacuum wave functions into the surface region produces surface emission, which contributes to the background cascade continuum intensity (Fig. 4), and is isotropic, i.e., produces the observed $\cos ^{2} \theta$ distribution.

Surface emission is responsible for the fact that the SEE spectral intensity is not zero within the regions $E_{G}$ identified with the energy gap extending throughout the bulk states Brillouin zone, Fig. 2 (cf. Fig. 3)。 This intensity can arise from only four possible sources: umklapp (elastic) scattering from bulk states throughout other regions of the Brillouin zone; thermal diffuse (quasielastic or phonon) scattering; inelastic electron-electron scattering during emission; and finally surface emission from any point at the metal-vacuum interface. The various bulk microscopic excitation processes: electron-hole pair, valence-hole Auger relaxation, and plasmon decay, merely serve to populate the one-electron states, and so cannot contribute to this intensity within the gaps in the SEE spectra.

Calculations, performed in extended $k$ space, ${ }^{20}$ have shown that, in general, umklapp scattering does not contribute significantly to the intensity in the gap regions for $\theta \lesssim 20^{\circ}$ directions [cf。columns (a) and (b), Figs. 6-8]. A similar argument holds against thermal diffuse scattering. Since the band gap $E_{G}$ extends throughout the Brillouin zone (Fig. $2)$, quasielastic (phonon) scattering from random regions throughout the zone will not contribute in this energy range. The region around $P H$ (Fig。2) is the only candidate volume of the zone, which the calculations ${ }^{20}$ and SEE spectra (Figs. 3-8) shown to contribute very little. Also, the fact that intensity is observed in the gap region from all three faces at all angles (albeit of differing magnitude) would indicate that its origin is a general characteristic of all three surfaces. Strong inelastic scattering of the excited electron during emission would be expected to smear-out one-electron 
band structure. However, the relative sharply defined band extremeties in the SEE spectra, Figs. 10 and 11 , show this not to be the case (for $E_{\mathrm{kin}}$ $\left.\lesssim \hbar \omega_{p}\right)$.

Therefore, we are left with emission from evanescent waves, highly localized in the solid at the excited surface atoms, i.e., surface emission, as the only possible source of this intensity at these energies. Directional photoemission measurements taken normal to the $\mathrm{W}(100)$ face also endorse this view ${ }_{0}^{16}$ Substantial intensity is again observed in the final states $\langle 100\rangle \mathrm{W}$ gap region $E_{G}$, which confirms transitions occurring to, and subsequent emission occurring from a "continuum" of surface evanescent states. The SEE results would indicate that similar photoemission intensity will be observed throughout the gap region on the (110) and (111) faces also. This is illustrated schematically for the simplest case of a nearly-free-electron metal in Fig. 12.

It is important to realize that $j_{s}(E)$ (i.e., surface emission) is a genuine surface electronic effect which is not confined to energies corresponding to bulk band gaps. The continuum distribution of vacuum-state plane waves are always able to "tail" a short distance into the surface of the metal, independent of the bulk band structure. Clearly, however, surface emission will be more apparent at energies corresponding to $\Delta E_{\text {gap }}$ regions. This is true for both photoemission [Fig. 12(a)] and secondary-electron-emission [Fig。12(b)] phenomena, although subtle differences will exist inherent in the photoexcitation matrix element which
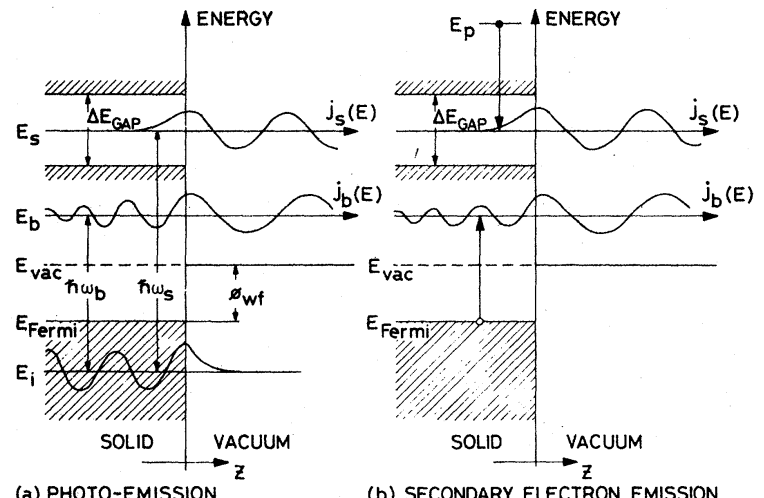

FIG. 12. Simple one-dimensional schematic illustration of unbound final-state electronic wavefunctions depicting bulk, $j_{b}(E)$, and surface, $j_{s}(E)$, current-density contributions for the case of (a) photoemission and (b) secondary-electron emission. Bulk states (energy $E_{b}$ ) and surface or band-gap states (energy $E_{s}$ ) above $E_{\text {vac }}$ can be populated (a) by photons (energy $\hbar \omega_{b}$ and $\hbar \omega_{s}$ ) or (b) via electron-hole pair transitions due to scattering between a primary electron (energy $E_{p}$ ) and a valence electron at or below $E_{\text {Fermi }}$; $\phi_{\text {wf }}$ is the work function.

couples initial (filled) and final (unfilled) states in the former case. ${ }^{18}$

\section{Transmission resonances}

Enhanced emission, arising from the quantummechanical (wave-matching) properties inherent in the transmission probability function $T\left(E, \overrightarrow{\mathrm{k}}_{\| 1}\right)$, will obviously complicate the analysis of electron emission spectra based solely on densities-ofstates arguments. The present results for the first time permit us to identify quite clearly the origin of transmission-induced spectral structure relating to the group velocity (i.e., transport) and wave matching at the surface (i.e., transmission) as determined by the energy band structure of the emitter. In the following discussion, we describe two quite distinct mechanisms which produce these transmission resonances. The first, and more general condition, ${ }^{20}$ relates to a vacuum-bulk-resonance-matching (VBRM) condition at points in $E-K$ space corresponding to almost perfect matching between the bulk crystal Bloch states, carrying current to the surface, and the plane-wave vacuum states. This is of special importance for those energy bands which determine emission from the $\mathrm{W}(111)$ surface, and, in particular, the peak $P$ together with the anomalous $\theta=0^{\circ}$ band-gap feature in the angle-resolved SEE spectra, Fig. 6 , referred to earlier. The second effect relates to a specific condition which arises for emission associated with a lower band-gap-edge resonance matching mechanism, which explains the anomalously high intensity and sharpness of peak $R$ in the $\theta=10^{\circ}, \mathrm{W}(100)$ spectrum, Fig。 8 .

\section{Vacuum-bulk-resonance matching}

We have already drawn attention to the fact that the $\mathrm{W}(111)$ spectra show overall much poorer agreement with the theoretical densities-of-contributing-states profiles than appears to be the case for the $\mathrm{W}(100)$ and $\mathrm{W}(110)$ surfaces. This can be seen by comparing the spectral intensity which appears at points $P, C, D$, and $F$ in curve $M$ with that predicted by the theoretical curve $T$ in the $\mathrm{W}(111) \theta=0^{\circ}$ spectrum, which for the sake of clarity, is reproduced in Fig.13. The gap $E_{G}^{\prime}$ is anomalous in that it is not predicted in the theoretical (umklapp contributions included) profile for $\theta=0$ emission [cf. curves $T$ and $M$, panel (b)].

The origin of the spectral anomalies circled in Fig. 13 can be understood by reference to Fig. 14 . It is possible, as illustrated in Fig. 14 for a simple one-dimensional nearly-free-electron case, to place free-electron parabolas (representing the dispersion relation of the emitted electron in vacuum) with their minima at the vacuum threshold in 


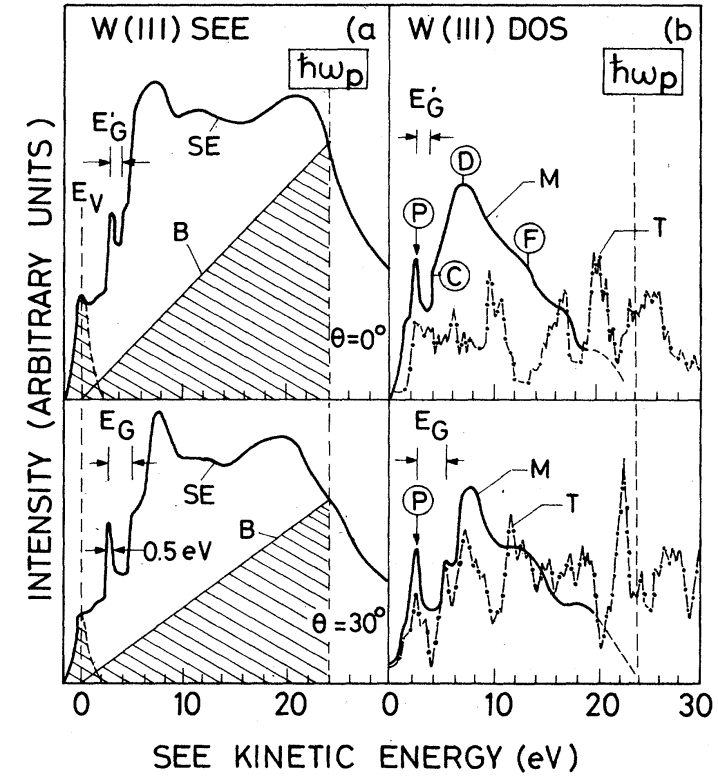

FIG. 13. Angle-resolved spectra of secondary electrons (SE) emitted in directions $\theta=0^{\circ}$ and $\theta=30^{\circ}$ with respect to the normal of a W(111) surface. Features in the measured or enhanced density-of-states (DOS) profiles [curves $M$, column (b)] which constitute "anomalies" when compared with the theoretical DOS profiles [curves $T$, column (b)] are shown circled.

such a way that they match exactly the actual band structure in energy as well as slope, for example at points $R_{L}$ and $R_{U}$ in the lower and upper bands, respectively, separated by the band gap $\left(E_{\text {gap }}\right)$ at the Brillouin-zone boundary $\frac{1}{2} G$. The actual case for the band structure of tungsten along the $\Gamma P$ and $P H$ symmetry directions of the bulk Brillouin zone, appropriate to the case of $\theta=0^{\circ}$ emission from a W(111) surface, is shown in Fig. 14(b). Vacuum-bulk-resonance-matching, between the bulk crystal Bloch states, carrying current to the surface and plane wave vacuum states, can occur at points $P, C, D$, and $F$ which coincide with the kinetic energies of the anomalous spectral features circled in Fig. 13(b). Any amount of normal momentum $K_{10}$ can be provided by the crystal lattice such that the minima of the free-electron parabolas can be placed at any point along the $K_{\perp}$ axis. The resonance peak $P$ which occurs at $E_{\mathrm{kin}} \simeq 3$ to $4 \mathrm{eV}$ is responsible for the apparent gap, $E_{\text {gap }}^{\prime}$, the origin of which has long been a puzzle in the $\langle 111\rangle$ symmetry-oriented-normal emission spectrum ${ }_{0}^{35}$ As illustrated in Fig。14(b), a gap is indeed expected if only the $\Gamma P$ symmetry direction in the Brillouin zone is considered, but the more relevant extended-zone scheme indicates that states along the zone edge line $P H$ should contribute to emission in this energy region. The enhancement

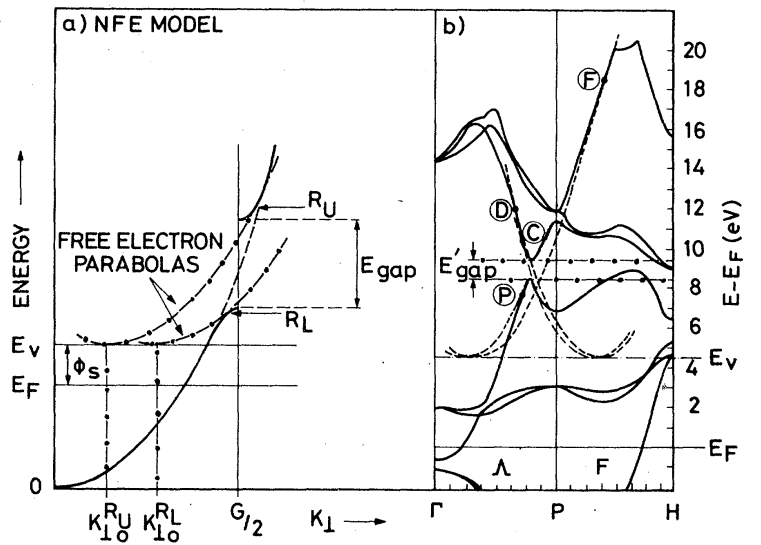

FIG. 14. Illustrating VBRM wave matching between free-electron parabolas and emitter energy bands for (a) the one-dimensional nearly-free-electron case and (b) the band structure of tungsten for emission in the $\langle 111\rangle$ direction $\left(\theta=0^{\circ}\right)$. The circled points indicate the origin of the spectral "anomalies" referred to in Fig. 13.

of emission due to the wave-matching condition at point $P$ (and $C$ ) produces an "apparent gap" which does not exist in the one-dimensional density-ofstates profile, Fig。13(b)。At $0=30^{\circ}$, the "true" gap is clearly identifiable in the density-of-states curve $T$, although the lower gap edge remains somewhat enhanced in the SEE spectrum due to transmission resonance effect, peak $P$ 。

These results illustrate that the transmission of current through the surface is considerably enhanced when the emitting state, $E_{f}(K)=E_{f}+E_{\mathrm{kin}}$, has a group velocity $\mid \nabla_{\overrightarrow{\mathrm{K}}}\left(E_{f}(\overrightarrow{\mathrm{K}}) \mid\right.$ which exactly equals that of the emitted electron in vacuum, ene rgy $E_{\mathrm{kin}}=\overrightarrow{\mathrm{p}}^{2} / 2 m$ 。 Under these particular conditions, the current carried by the Bloch waves incident on the surface is conserved such that the reflected component waves are effectively zero, i.e., the transmission probability amplitude coefficients reach a maximum value. Conservation of energy and parallel momentum, $\hbar^{-1} \overrightarrow{\mathbf{P}}_{\mathbf{H}}$ $=\overrightarrow{\mathrm{k}}_{11}+\overrightarrow{\mathrm{G}}_{\|} \equiv \overrightarrow{\mathrm{K}}_{\| 1}$, yields the relationship $\hbar \overrightarrow{\mathrm{K}}_{\| 1}=\overrightarrow{\mathrm{P}}_{\|}$ $=\sin \theta\left(2 m E_{\mathrm{kin}}\right)^{1 / 2}$, so that in a simple free-electron model $E_{\mathbf{k i n}}=\left(\hbar^{2} / 2 m\right)\left|\overrightarrow{\mathrm{K}}_{\|}^{2}+\overrightarrow{\mathrm{K}}_{\perp}^{2}\right|-E_{v}$ and

$$
\overrightarrow{\mathrm{K}}_{\perp}^{2}-\left(\overrightarrow{\mathrm{K}}_{11} / \tan \theta\right)^{2}=2 m E_{v} / \hbar^{2},
$$

i.e., the $\overrightarrow{\mathrm{K}}$-space curve relating to a given emission angle $\theta$ is an hyperbola with $\overrightarrow{\mathrm{K}}_{11}=\overrightarrow{\mathrm{K}}_{1} \tan \theta$ as asymptote. The above VBRM points occur at locations on the energy bands in $\overrightarrow{\mathrm{K}}$ space whose slopes are tangential to this asymptote。Detailed $\overrightarrow{\mathrm{K}}_{1}$ vs $\overrightarrow{\mathrm{K}}_{\| 1}$ space plots are given in a separate theory paper $^{20}$ which show that such effects are not restricted to the symmetry-oriented-normal emission geometry。Transmission-enhanced structure, such as the points circled in Fig. 13 is of general 
occurrence for $\mathrm{W}(111)$ emission due to the dispersion of the bulk energy bands in the corresponding regions of the Brillouin zone. Notably peak $P$ is predicted for all off-normal directions, as is observed to be the case (Fig. 6), whereas the intensity enhanced feature $D$ (Fig. 13) is predicted for $\theta \lessgtr 50^{\circ}$ only and is observed to disappear from the spectra for larger angles. Similar arguments explain features $C$ and $D$ (Fig. 13) and are detailed in a separate paper..$^{20}$

Attention is also brought to the fact that it is easy to confuse the behavior of features such as peak $P$ in the $\mathrm{W}(111)$ spectra with that of a surface resonance band similar to peak $S$ in the $\mathrm{W}(100)$ spectra discussed earlier (Sec. III C). This is seen by comparing the angular dependence of the normal-processes gap ( $\left.E_{G}, \mathrm{Fig} .6\right)$ with the dispersion behavior of peak $P$ as a function of $\theta=\sin ^{-1}\left[\hbar \overrightarrow{\mathrm{K}}_{11}\right]$ $\left.(2 m E)^{1 / 2}\right]$, Fig. 15. Peak $P$ appears to extend out of the lower band edge, $\vec{k}_{11}=0.5 \AA^{-1}$ at $\theta=30^{\circ}$, and evolves into the gap region along the $\bar{T}$ SBZ symmetry line (inset). (The polar-angle measurements were not able to extend out to the SBZ boundary, $4 \sqrt{2} \pi / 3 a \simeq 1.75 \AA^{-1}$ for this face.) In this case, however, the actual gap $E_{G}$ is ill defined as seen by the complex nature of the umklapp contributions of bulk states appearing in this energy re-

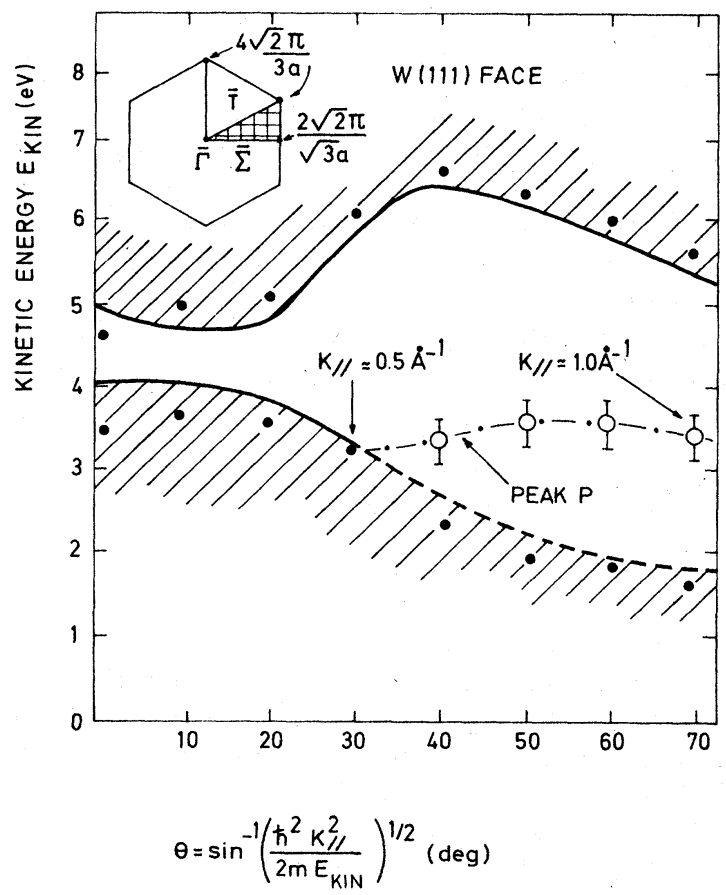

FIG. 15. Angular dependence of the SEE spectral peak $P$, in relation to the reduced-zone band edges indicated as $E_{G}^{\prime}$ (Fig.6), as a function of polar angle $\theta=\sin ^{-1}\left[\hbar \vec{k}_{11}\right]$ $\left.2 m E_{\text {kin }}\right)^{1 / 2}$ ] along the $T$ direction of the W(111) surface Brillouin zone (inset). gion, $40^{\circ} \leqslant \theta \leqslant 70^{\circ}$ [column b, Fig.6]. For this reason, the lower band edge is shown dashed in Fig. 15. The important point here is that the origin of this spectral structure relates to emission from bulk rather than surface states. Also, and more important, due to the wave-matching determinant nature of the transmission probability function $T\left(E, \vec{k}_{\| 1}\right)$, this structure is particularly sensitive to surface contamination. ${ }^{45}$

\section{Lower-band-edge-resonance matching}

In any physical system, a sharp resonance is the result of constructive interference between component waves of comparable magnitude. Just such a situation exists at the extremities of energy band gaps at the zone boundary (cf. $\frac{1}{2} \vec{G}$, Fig. 14) where the component plane waves travelling in opposite directions combine to form standing waves:

$$
\Psi( \pm)=\exp (i \pi Z / a) \pm \exp (-i \pi Z / a),
$$

where $a$ is the lattice parameter in the $z$ direction normal to the surface. Above the vacuum level, the vacuum plane waves are able to match with the above band-edge standing waves so as to extend the pinning point out to the detector. The resonance is thereby transferred out of the surface, as illustrated in Fig.16. Secondary electrons cascading down in energy serve as the current source. Moreover, the lower $E_{G}$ edge is associated with the $\Psi(+)=2 \cos (\pi Z / a)$ component representing a maximum in the standing wave amplitude at the outermost atomic layer, i.e., the probability density distribution $\beta(+)=|\Psi(+)|^{2} \propto \cos ^{2}(\pi Z / a)$ which effectively piles up electronic charge right at the interface boundary, thereby providing a local source of current. In contrast, the upper edge of

(a)

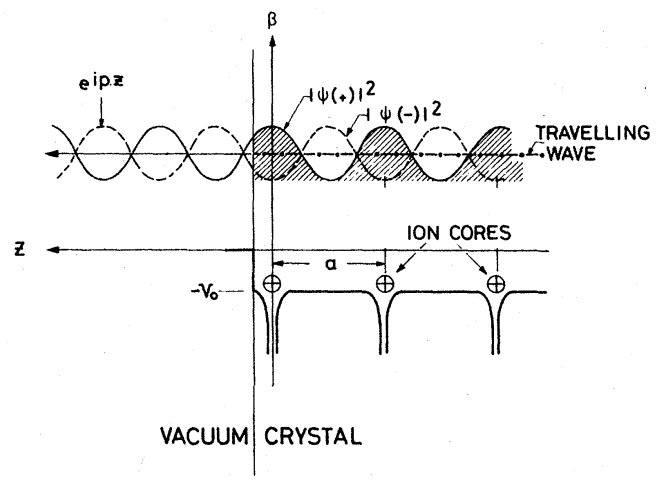

FIG. 16. Schematic illustration of (a) the probability density distribution of electrons $\beta=|\Psi( \pm)|^{2}$ in the surface region for the two standing waves $\Psi(-) \pi \sin (\Pi Z / a)$ and $\Psi(+) \pi \cos (\Pi Z / a)$ at upper and lower extremities of an energy band gap at a zone boundary; (b) the associated one-dimensional (muffin-tin) potential spatial distribution of the ion cores for $Z$ normal to the surface. 
the gap is determined by the $\Psi(-)=2 i \sin (Z / a)$ standing wave such that $\beta(-)=|\Psi(-)|^{2} \propto \sin ^{2}(\pi Z / a)$ distributes electronic charge preferentially between the outermost and penultimate layers, and represents a minimum in the wave amplitude at the surface. Surface emission (Sec. IIIC) arising from the tailing of the plane vacuum state into the solid will be enhanced more, therefore, by the $\Psi(+)$ (lower-edge) condition.

Peak $R$, which arises for emission at $\theta=10^{\circ}$ from the $\mathrm{W}(100)$ face (Fig.8), represents a striking example; the emission is sharply peaked at the lower band edge, and represents an enhancement of the theoretical state densities. A similar situation exists for peak $P$ for emission at $\theta=20^{\circ}$ from the $\mathrm{W}(111)$ face (Fig. 6), but here the effect is less clear due to the simultaneous incidence of the above VBRM mechanism. ${ }^{20}$ Nevertheless, in both of these cases, the spectral structure is very sharply resolved with a half width of the order of $0.5 \mathrm{eV}$. The two cases are compared in Fig。 17 and labeled $R$. More importantly, such features have only been seen at the lower and not the upper extremities of the bulk energy gap, identified as $E_{G}$ (Fig. 17), which would endorse the extremely surface localized nature of the process. Again also, these features are sensitive to surface perfection and contamination effects. ${ }^{45}$

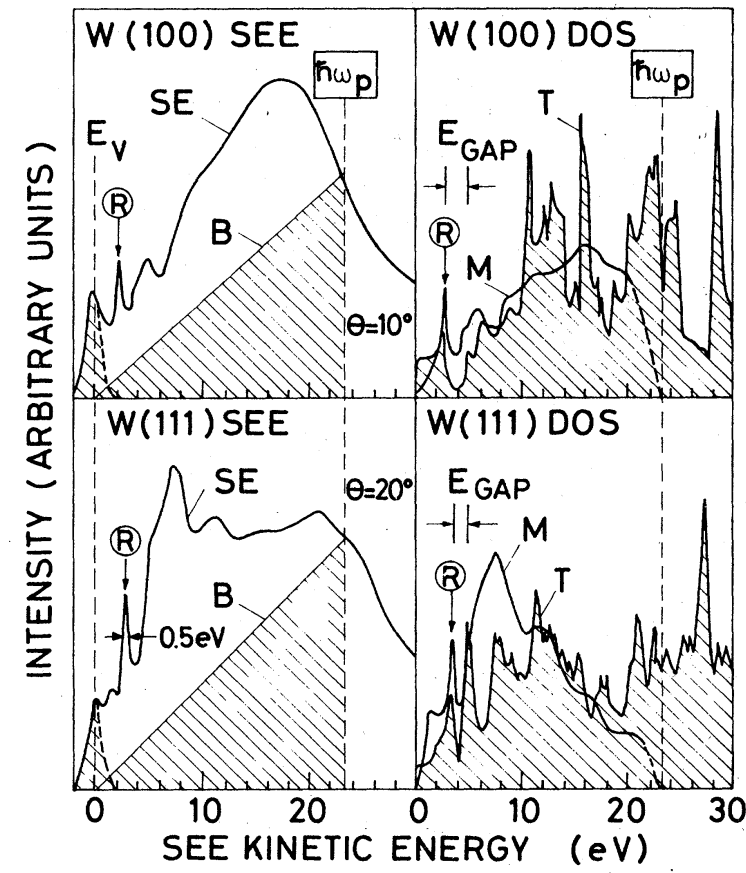

FIG. 17. Angle-resolved spectra of secondary electrons (SE) emitted in directions $\theta=10^{\circ}$ for $\mathrm{W}(100)$ and $\theta=20^{\circ}$ for $\mathrm{W}(111)$ showing the incidence of extremely sharp resonances $R$ appearing at the lower edge of the band gap $E_{G}$.

\section{CONCLUDING REMARKS}

As stated in the Introduction, a specific aim of the present work was to establish the viability of angle-resolved secondary-electron-emission spectroscopy as a means for investigating the high-energy band structure of solids. Much previous theoretical work ${ }^{5-8,30}$ has tended to concentrate on the detailed mechanism of the initial interaction between the incident electron and the valence electrons in the solid. Experimental effort ${ }^{1-4}$ searched for diffraction effects in directions defined by the reciprocal-lattice $\vec{G}$ vectors supporting the interband transitions. This approach, together with the fact that calculations were restricted to free-electron-gas models of the solid, has tended to retard the development of SEE as a tool for band-structure studies. The angle-resolved SEE profiles are in fact rich in structure relating to the detailed electronic properties of the metal, as the results here have shown.

The present work has concentrated on correlating the observed fine-structure with states predicted within the single-particle approximation. The theoretical and experimental bulk states profiles show convincing agreement, particularly as regards the position and behavior with angular variation of the predicted energy band gap around $E=9 \mathrm{eV}$ above $E_{F}$ in tungsten. The problem of the "cascade peak" background curve is indicative of a future requirement for wave-matching calculations so as to include not only bulk states propagating Bloch waves but also, the so-called surface evanescent waves. The present results have shown that the overall secondary-electron yield, rather than being represented as a sum of various separate microscopic excitation mechanisms as has often been the approach to date, ${ }^{1-9,30}$ is better represented as the sum of basically two contributions:

$$
J_{\mathrm{SEE}}^{\text {total }}=\int_{0}^{\pi} d \Omega \int_{0}^{E_{\max }} j_{\mathrm{SEE}}(E, \Omega) d E \equiv J_{\mathrm{SEE}}^{\text {surface }}+J_{\mathrm{SEE}}^{\text {bulk }} .
$$

The bulk contribution represents emission from the "infinite crystal" Bloch states (including umklapp effects) which propagate out from the bulk crystal. Separate contributing "surface" processes may be distinguished:

$$
J_{S E E}^{\text {surface }}=J_{S E E}^{S S}+J_{S E E}^{S E},
$$

$J_{S E E}^{S S}$ representing emission from bands of evanescent surface states (resonances) with $\vec{k}_{||}$periodic in the surface lattice and $\vec{k}_{1}$ complex, the wavefunction amplitude decaying exponentially away from the metal surface. These states may be in- 
trinsic or extrinsic (adsorbate induced), and produce emission at energies corresponding to the bulk states energy gaps, contributing band-gap SEE. They form bands of states which can propagate only in the surface. $J_{\mathrm{SEE}}^{S E}$ represents surface emission, arising out of the fact that there is always a finite probability than the plane-wave state, describing an electron arriving at the detector, can tail a short distance into the metal surface representing a local source of current due to surface atoms excited to energies above the vacuum level. These conditions exist for all $\vec{k}_{\|}$in the surface Brillouin zone such that the emission is isotropic in angle giving the observed $\cos ^{2} \theta-$ dependent background intensity distribution.

While it may be phenomenologically useful, however, to make this distinction between separate contributing bulk and surface effects, the incidence of transmission resonances are a clear indication that the current density function is not a simple sum of separable emission processes. Bulk and surface electronic effects are intrinsic properties of the amplitude of the final-state incoming wave function at the surface and its penetration into the metal. Surface resonance bands and surface spectral densities-of-states effects serve to enhance the amplitude of the incoming wave as it passes through the surface, thus representing a finite intensity enhancement in the differential current at the detector. The composition of the final-state wave function itself is a consequence of wave matching between the various incident and scattered component waves summed over the whole surface Brillouin zone. For this reason, the amplitudes of the final-state waves at the metal-vacuum interface reflect an "average surface localdensity-of-states distribution.,"23 The observation of bulk states features in the electron emission spectra is in fact a direct consequence of the quantum-mechanical wave-matching conditions . ${ }^{20}$

The incidence of transmission resonances may be regarded therefore as scattering resonances arising from (coherent) interference effects between the incident and scattered components of the Bloch waves incident on the solid-vacuum boundary. That is, wave matching relates not only to the amplitudes but also to the phase of the component waves transmitted and reflected at the surface. The importance of such phase-determinant (as opposed to amplitude-determinant) resonances in the transmission probability have been postulated in LEED intensity analysis. ${ }^{46}$ The amplitude coefficients of low-energy electron reflection, $R_{n k}(E)$ $=\left|1-T_{n k}(E)\right|$, are complex numbers each of which characterize the reflection intensity $\left|R_{n k}(E)\right|^{2}$ and phase, $\arg \left|R_{n k}(E)\right|$, of the plane wave incident on the surface. Inelastic scattering (absorption) re- quires that the electron energy (or the surface normal component of the propagation vector $\overrightarrow{\mathrm{K}}_{1}$ ) has complex values. For a real scattering potential, McRae ${ }^{46}$ has shown that singularities occur in the complex amplitude function which relate to the band structure of the substrate crystal. For a clean unrelaxed crystal surface, the extremities of band gaps are characterized by crystal branch points, which lie on the real $\overrightarrow{\mathrm{K}}$ axis taken along the appropriate one-dimensional section of the substrate band structure. At such points, the imaginary part of the amplitude vanishes and sharp resonances are predicted in the reflection (transmission) coefficients.Surface states (resonances) are a consequence of poles in the complex amplitude function arising out of the scattering potential at the surface being different in magnitude (or spatial location) to that in the bulk. Similar effects to those described in Secs. III C and III D are therefore expected in (low-) energy electron-reflectivity analysis, i.e., with structure in the $1-R_{n k}(E)$ profiles. Such measurements as have been carried out to date on tungsten single crystals serve to endorse this view. ${ }^{42,47,48}$ An important distinction, however, is that whereas electron-reflectivity intensity measurements observe signal variations in the backscattered current, SEE involves much stronger foreward scattering directly from a continuum source of unbound states in the crystal..$^{49}$

In relation to ultraviolet photoemission, angleresolved SEE spectroscopy provides a valuable method for investigating the final states $|f\rangle$ decoupled from photoexcitation from the initial states $|i\rangle$. In the former case, the energy of the escaping electron will reflect both final electron state and initial hole state (i.e., dynamic screening and Auger relaxation) lifetime effects. In cases in which the initial states's bands are relatively flat such that the hole state remains localized (i.e., high effective mass), then the photoemitted electron will exhibit a relaxation shift in energy..$^{50}$ Also, the coupling of $|i\rangle$ and $|f\rangle$ via the optical transition matrix element will produce related momentum broadening $\Delta K_{n \leq}$ in the photoemission spectral line shapes. In contrast, the SEE spectra only reflect final electron states's lifetime broadening. The present results would indicate this, together with any self-energy shifts to be less than $\pm 0.2 \mathrm{eV}$ for energies $E_{\mathrm{k} \text { in }} \leqslant \hbar \omega_{p}$ the bulk plasmon resonance (see Ref $。 20$ )。 This realization would indicate that a comparison of SEE and photoemission spectral features, in a comparable energy range, to be a useful method for separating and investigating these various effects in different materials.

The "anomalous" SEE spectral structure due to resonances in the transmission probability function 
will also be manifest in photoemission spectra. Such effects can easily be confused with an optical response or intrinsic densities-of-states origin whereas, in reality they constitute a complex finalstate modulation effect. The incidence of transmission resonances in photoemission spectra is likely, however, to be less than in SEE in view of the restrictions imposed by any particular (fixed) photon frequency, which would appear to be the case to date ${ }^{51,52}$ Transmission effects will become more prevalent, however, in cases in which the final states are scanned continuously by the photon energy (such as is the case in constant-initial-state spectroscopy ${ }^{53}$ )。

As a final concluding remark, in answer to the question as to "why the SEE fine structure should appear so sharply resolved for tungsten?," the reason relates to the unusual forbidden gap around $E \simeq 9 \mathrm{eV}$ above $E_{F}$ which effectively makes this metal a final-state "insulator," i.e., hot electrons in states above this gap find it difficult to decay down to lower energies by electron-hole pair scattering and hence, have unusually long lifetimes. This is fortunate since it allows us a wider range of energies to probe the electronic properties than might be the case with other metals.

\section{ACKNOWLEDGMENTS}

The authors wish to express their gratitude to colleagues in the Surface Physics Group, ESTEC, particular M. R. Barnes for valuable technical assistance, Dr.B. Feuerbacher for many hours of rewarding scientific discussion, and $\mathrm{Dr} . \mathrm{B}$. Fitton for continuing support.Also, Dr。R. A. Armstrong of the Canadian National Research Council for communicating unpublished electron-reflectivity results, and Professor J. M. Blakely of Cornell University for supplying us with tungsten single crystals. This work has partly been supported by the Danish Natural Science Research Council.
${ }^{1}$ A. J. Dekker, in Solid State Physics, edited by F. Seitz and D. Turnbull (Academic, New York, 1958), Vol. 6, p. 215; O. Hachenberg and W. Brauer, in Advances in Electronics and Electron Physics, edited by L. Marton (Academic, New York, 1959), Vol. 11, p. 413.

${ }^{2}$ H. L. H. Jonker, Philips Res. Rep. 12, 249 (1957).

${ }^{3} \mathrm{~J}$. Burns, Phys. Rev. 119, 102 (1960), and references therein.

${ }^{4}$ G. Appelt, Phys. Status Solidi 27, 657 (1968).

${ }^{5}$ A. van der Ziel, Phys. Rev. 92, 35 (1953).

${ }^{6} \mathrm{P}$. A. Wolff, Phys. Rev. 95, $\overline{56}$ (1954).

${ }^{7}$ H. W. Streitwolf, Ann. Phys. 3, 183 (1959).

${ }^{8}$ H. Stolz, Ann. Phys. 3, 197 (1959).

${ }^{9}$ G. F. Amelio, J. Vac. Sci. Technol. 7, 593 (1970).

${ }^{10}$ E. J. Scheibner and N. N. Sharp, Surf. Sci. ㅈ, 247 (1967).

${ }^{11}$ M. P. Seah, Surf. Sci. 17, 132 (1969).

${ }^{12} \mathrm{~J}$. J. Lander, Phys. Rev. 91, 1382 (1953).

${ }^{13}$ R. F. Willis, B. Feuerbacher, and B. Fitton, Phys. Lett. A 34, 231 (1971); Phys. Rev. B 4, 2441 (1971).

${ }^{14}$ R. F. Willis, B. Fitton, and G. S. Painter, Phys. Rev. B 9, 1926 (1974).

${ }^{15}$ R. F. Willis, Phys. Rev. Lett. 34, 670 (1975); in Proceedings of the Fourth International Conference on Vacuum Ultraviolet Radiation Physics, edited by E. E. Koch, R. Haensel, and C. Kunz (Pergamon, Vieweg, 1974), p. 582 .

${ }^{16} \mathrm{~B}$. Feuerbacher and B. Fitton, Phys. Rev. Lett. 30, 923 (1973); B. Feuerbacher and N. E. Christensen, $\overline{\text { Phys. }}$ Rev. B 10, 2373 (1974).

${ }^{17}$ In this work we wish to distinguish between the following: the number of states in an energy range $E$ to $E+d E$ for all allowed $\overrightarrow{\mathrm{k}}$ vectors, expressed as the usual surface integral with respect to surfaces of constant energy $S(E)$ in $\overrightarrow{\mathrm{k}}$ space, is

$$
N(E) d E=\int_{k} \frac{d S}{4 \pi^{3}\left|\nabla E_{\overrightarrow{\mathrm{k}}}(\overrightarrow{\mathrm{k}})\right|} d E,
$$

where $\nabla E_{\overrightarrow{\mathrm{k}}}(\overrightarrow{\mathrm{k}})$ is a vector normal to $S(E) ; j_{\mathrm{SEE}}(E, \Omega)$, however, places some restriction on $k$-vector selection so that we define $N(E) d E \equiv D(E) d E$ as the one-dimensional distribution function along a symmetry line in $k$ space. We also define a local density of states function:

$$
\rho_{0}(\overrightarrow{\mathrm{r}}, E)=\sum_{n} \delta\left(E-E_{n}\right)\left|\Psi_{n}(\overrightarrow{\mathrm{r}})\right|^{2},
$$

for an eigenfunction of the system $\psi_{n}(\overrightarrow{\mathrm{r}})$ and associated eigenvalue $E_{n}$, writing $\rho_{0}(\overrightarrow{\mathrm{r}}, E) \equiv \rho_{B}(\overrightarrow{\mathrm{r}}, E)$, in the bulk crystal and $\rho_{0}(\overrightarrow{\mathrm{r}}, E) \equiv \rho_{S}(\overrightarrow{\mathrm{r}}, E)$ at the surface. These distinctions are necessary since $j_{\mathrm{SEE}}(E, \Omega)$ contains current from both Bloch functions propagating along specific directions through the surface from the bulk plus contributions from states spacially localized at the surface.

${ }^{18}$ R. F. Willis and N. E. Christensen, in Proceedings of the International Symposium on Photoemission, Noordwijk, Holland, September, 1976 , edited by R. F. Willis et al., European Space Agency Publ. No. SP 118, REV 1 (Scientific and Technical ESTEC, Noordwijk, Holland, 1976).

${ }^{19}$ N. E. Christensen and B. Feuerbacher, Phys. Rev. B 10, 2349 (1974).

20 N. E. Christensen and R. F. Willis, J. Phys. C (to be published).

${ }^{21}$ P. J. Feibelman and D. E. Eastman, Phys. Rev. B 10, 4932 (1974)。

${ }^{22}$ I. Adawi, Phys. Rev。134, A788 (1964).

${ }^{23}$ See, for example, A. Modinos and N. Nicolaou, Phys. Rev. B 13, 1536 (1976); T. E. Feuchtwang and P. H. Cutler, $\overline{i b} i d .14,5237$ (1976).

${ }^{24}$ R. F. Willis and M. R. Barnes, Rev. Sci. Instrum. (to be published).

${ }^{25}$ This is not the case, for example, in a strongly anisotropic material such as graphite (Refs. 13 and 14) where both the energy-loss profiles and the SEE energy distribution is found to be dependent on the orienta- 
tion of the incident primary-electron beam (and hence, on the scattered wave vector $\vec{q}$ ). No such effects have been observed in the present work for tungsten due to its cubic crystal structure (see text).

${ }^{26}$ R. F. Willis, Farad. Disc. Chem. Soc. 60, 245 (1975); R. F. Willis, B. Feuerbacher, and B. Fitton, Solid State Commun. 18, 185 (1976).

${ }^{27} \mathrm{See}$, for example, J. C. Tracy and J. M. Blakely, Surf. Sci. 15, 257 (1969).

${ }^{28}$ L. F. Mattheiss, Phys. Rev. 139, A1893 (1965); I. Petroff and C. F. Viswanathan, Phys. Rev. B $\underline{4}, 799$ (1971).

${ }^{29}$ P. Soven, E. W. Plummer, and N. Kar, Soli State Sci. 6, 109 (1976).

${ }^{30}$ M. S. Chung and T. E. Everhart, J. Appl. Phys. 45, 707 (1974).

${ }^{31}$ R. M. Stern and A. Gervais, Surf. Sci. 17, 273 (1969).

${ }^{32} \mathrm{H}$. Raether, in Springer Tracts in Moder $m$ Physics, edited by G. Höhler (Springer-Verlag, Berlin, 1965), Vol.38, p. 84.

${ }^{33}$ J. H. Weaver, C. G. Olson, and D. W. Lynch, Phys. edited by G. Höhler (Springer-Verlag, Berlin, 1965), Vol. 38, p. 84.

tropic in angle for these "background" electrons, a linear variation with kinetic energy $E_{\text {kin }}$ of the emitted current has been found to be a quite good approximation for the behavior of several metals near threshold [R. W. Strayer, W. Mackie, and L. W. Swanson, Surf. Sci. 34, 225 (1973)]. This is true since the fast moving secondary electrons in final states above the vacuum level effectively do not "see" the shape of the potential barrier at the surface, which would affect this linearity otherwise.

${ }^{35}$ R. F. Willis and B. Feuerbacher, Surf. Sci. 53, 144 (1975).

${ }^{36}$ Refraction at the surface prevents umklapp contributions from appearing at the lower kinetic energies $E_{\text {kin }} \lesssim 10 \mathrm{eV}$ in the normal direction of emission [Ref. 20 and P. O. Nilsson and L. Ilver, Solid State Commun. 18, 677 (1976)]. The symmetry-oriented geometry for emission normal to the crystal surface is particularly attractive for angle-resolved photoemission spectral analysis for this reason [Ref. 16 and P. Heimann et al. Phys. Rev. Lett. 37, 775 (1976)].

${ }^{37}$ G. F. Koster, in Solid State Physics, edited by F. Seitz and D. Turnbull (Academic, New York, 1965), Vol. 5, Chap. 3.

${ }^{38}$ B. D. Barford and R. R. Rye, J. Vac. Sci. Technol. 9 , 673 (1972).

${ }^{39}$ The probability amplitude of an electron in such a surface state decreases exponentially with distance from the surface (inside and outside) and is "nonlocalized" in that the electron may be found anywhere on the surface. However, the electron can never be completely trapped at the surface for energies above the vacuum level, since it can always match into a plane wave in vacuum. The formal expression is that the surface state is a resonance to distinguish it from the usual bound states.

${ }^{40}$ N. V. Smith and L. F. Mattheiss, Phys. Rev. Lett. 37, 1494 (1976).

${ }^{41}$ R. F. Willis, B. Feuerbacher, and N. E. Christensen, Phys. Rev。 Lett. 38, 1087 (1977).

${ }^{42} \mathrm{~A}$ similar resonance feature is observed in electron reflectivity (i.e., LEED) intensity measurements [E. G. McRae, Phys. Rev. B 16, 4718 (1977)] and relates to resonance scattering between the topmost layer of $\mathrm{W}$ atoms and the substrate crystal. The origin may be traced to atomic $d$-wave (phase-dependent) scattering among the $\mathrm{W}$ atoms in the outermost monolayer [P. J. Jennings and E. G. McRae, Surf。Sci. 23, 363 (1970)].

${ }^{43}$ R. F. Willis, B. Feuerbacher, and B. Fitton, Solid State Commun. 18, 1315 (1976); for a review see E. W. Plummer, in Topics in Applied Physics, edited by R. Gomer (Springer-Verlag, Berlin, 1975), Vol. 4, p. 143.

${ }^{44}$ V. Heine, Proc. Phys. Soc. Lond. 81, 300 (1962).

${ }^{45}$ Experimentalists, up until recently, and particularly in photoemission studies, have usually employed sensitivity to gas adsortption as a criterium for identifying emission from surface states, together with insensitivity of the peak positions to photon energy and sensitivity to polarization effects. These criteria have recently been questioned however in the light of studies on bulk states behavior in energy band-gap regions (see, for example, J. Lecante et al. in Ref. 18; C. Noguera et al., Phys. Rev. Lett. 38, 1171 (1977)). The incidence of transmission resonances in the final-state wave will certainly produce surface sensitive spectral features whose origin is related to the bulk band structure.

${ }^{46}$ E. G. McRae, Surf. Sci. 47, 167 (1975).

${ }^{47} \mathrm{R}$. A. Armstrong, Surf. Sci. 47,555 (1975); C.an. J. Phys. 44, 1753 (1966). See also R. F. Willis, Farad. Disc. Chem. Soc., 60, 245 (1975).

${ }^{48} \mathrm{E}$. G. McRae, J. M. Landwehr, and C. W. Caldwell, Phys. Rev. Lett. 38, 1422 (1977).

${ }^{4 y}$ R. F. Willis, Inst. Phys. Lond. Conf. Ser. 41,233 (1978).

${ }^{50}$ J. W. Gadzuk, Phys. Rev. B 14, 2267 (1976).

${ }^{51}$ D. J. Spanjaard, D. W. Jepsen, and P. M. Marcus, Phys. Rev. B 15, 1728 (1977).

${ }^{52}$ N. E. Christensen and R. F. Willis, Solid State Commun. 25, 721 (1978).

${ }^{53} \overline{\mathrm{R}}$. J. Smith, J. Anderson, J. Hermanson, and G. J. Lapeyre, Solid State Commun. 19, 975 (1977); see also Ref. 18 for a discussion of this point. 